\title{
The meteor-shower complex of 96P/Machholz revisited
}

\author{
L. Neslušan, Z. Kaňuchová, and D. Tomko
}

\begin{abstract}
Astronomical Institute, Slovak Academy of Sciences, 05960 Tatranská Lomnica, Slovakia
e-mail: [ne;kanuchova;dtomko]@ta3.sk
\end{abstract}

Received 28 August 2012 / Accepted 8 January 2013

\begin{abstract}
Aims. The structure of the complex of meteoroid particles released from comet 96P/Machholz is studied to reveal a relationship among the meteor showers observed in the Earth's atmosphere that belong to this complex.

Methods. For eight perihelion passages of the parent comet in the past, we model theoretical streams associated with comet 96P and follow their dynamical evolution until the present. Subsequently, we analyze the orbital characteristics of the streams, especially of their parts approaching the Earth's orbit.

Results. The dynamics of the stream is controlled by Jupiter, which changes the initial orbits of the particles into the orbits situated within several specific corridors. It thus creates a filamentary structure of the complex. Six filaments approach the orbit of the Earth producing four well-known meteor showers and two showers, whose identification with $\kappa$-Velids and $\alpha$-Cetids is not certain. The known showers, in order of the predicted abundance of meteors, are daytime Arietids, Southern $\delta$-Aquarids, Quadrantids, and Northern $\delta$-Aquarids. The filaments corresponding to the Arietids, $\delta$-Aquarids $\mathrm{S}$ and $\mathrm{N}$, and possibly $\alpha$-Cetids constitute the ecliptical component and those corresponding to the Quadrantids and possibly $\kappa$-Velids constitute the toroidal component of the complex.
\end{abstract}

Key words. comets: individual: 96P/Machholz - meteorites, meteors, meteoroids

\section{Introduction}

The dynamical evolution of the meteoroid stream of comet 96P/1986 J2 (Machholz 1) is studied within our series of studies of comets in orbits that are situated relatively far from the orbit of the Earth (Neslušan 1999; Tomko \& Neslušan 2010). We have already studied the part of the 96P stream related to the Quadrantid meteor shower (Kaňuchová \& Neslušan 2007). Since not only Quadrantids, but also several filaments of 96P stream cross the Earth's orbit, we repeat our earlier analysis to provide, as far as possible, the complete picture of meteor showers associated with this comet.

When taking the dynamics controlled by Jupiter and the short orbital period of 5.24 years into account, 96P/Machholz can be regarded as the Jupiter-family comet even though its Tisserand parameter with respect to the Jupiter is slightly smaller than 2 at 1.942 . Among the short-period comets, 96P is exceptional for the remarkably short perihelion distance of its orbit, $q=0.124617662286515 \pm 0.00000021044$ AU. The other elements characterizing its orbit are: eccentricity $e=$ $0.958684989318405 \pm 0.000000073526$, argument of perihelion $\omega=14.61824824802251^{\circ} \pm 0.00002832^{\circ}$, longitude of ascending node $\Omega=94.55063039982306^{\circ} \pm 0.000018413^{\circ}$, inclination $i=59.9551168902691^{\circ} \pm 0.000069022^{\circ}$, and Julian date of the perihelion passage $T(\mathrm{JDT})=2454195.122101750695 \pm$ 0.000013656 days for epoch JDT $=2454205.5$ (JPL Small-Body Database Browser ${ }^{1}$ ).

The comet was mentioned for the first time in the context of meteor astronomy in 1990 by McIntosh, who suggested that it might be the parent body of the Quadrantid meteoroid stream. According to McIntosh, the perturbation behavior of 96P is similar to that of the Quadrantid stream except that their 4000-year cycles are shifted by 2000 years. In addition, he noticed that the

\footnotetext{
1 http://ssd.jpl.nasa.gov/sbdb.cgi
}

orbital evolution of the $\delta$-Aquarid and Arietid meteor streams were also consistent with this behavior, except for a different phase shift.

The individual meteor streams of the 96P complex have already been studied before the discovery of this comet. Williams, Murray, and Hughes (Williams et al. 1979; Hughes et al. 1979) integrated ten test particles in the orbits of Quadrantids and already in that time they revealed the large reduction of their inclination (down to $12^{\circ}$ ) and perihelion distance (down to $0.1 \mathrm{AU}$ ), as well as further evolutionary characteristics.

Froeschle \& Scholl (1986) investigated the dynamics of the Quadrantids on a timescale of $10^{3}$ years. They found that the stream splits into filaments with distinctly different dynamical evolution. They pointed out the situation of the stream orbits being close to the 2:1 resonance with Jupiter.

The complicated structure of the Quadrantids and their eventual relationship to the $\delta$-Aquarids was noticed by Babadzhanov \& Obrubov (1992). Babadzhanov et al. (1991) revealed that the stream, part of which are the Quadrantids and another part the $\delta$-Aquarids, intersects the Earth's orbit four times, and each of these parts can have both northern and southern strands. Besides the Quadrantids and Northern and Southern $\delta$-Aquarids, they pointed out the relationship with the Ursids and Carinids. Babadzhanov and Obrubov developed their study further after the discovery of 96P and its relation with the Quadrantids (see further discussion below).

Wu \& Williams (1992) integrated the orbits of Quandrantids, selected from the IAU MDC data, from the present to 7000 years in the past and found the groups of particles with the variation of orbits typical of the 96P meteor complex. Except for these variations, some orbits were behaving chaotically and some remained in the current orbital corridor of the Quadrantids for the entire integration period. However, the authors did not identify the alternative filaments with the known non-Quadrantid streams. 
The complex studies of the whole 96P stream started after the discovery of comet $96 \mathrm{P} / \mathrm{Machholz}$ in 1986 and the recognition by McIntosh (1990) that this comet can be the parent body of the Quadrantids. McIntosh alone was reluctant to identify comet 96P as the single parent body of Quadrantids and preferred to consider both the comet and the stream to be members of a larger family of interplanetary bodies.

As mentioned above, Babadzhanov \& Obrubov $(1992,1993)$ went on with their study of the $96 \mathrm{P}$ complex. They predicted eight meteor showers related to 96P: Quadrantids, Ursids, Carinids, $\kappa$-Velids, Northern and Southern $\delta$-Aquarids, Daytime Arietids, and $\alpha$-Cetids. They found two of these showers, Carinids and $\kappa$-Velids, in three radio-meteor catalogs created from the observations of the southern sky.

Simultaneously, Jones \& Jones (1993) investigated the stream of $96 \mathrm{P}$ by integrating 500 test particles representing the stream. They concluded that if the comet was captured at its last close approach with Jupiter about 2200 years ago, then there has been enough time for the resulting stream to produce most of the features of the presently observed Quadrantid/Arietid/Southern $\delta$-Aquarid complex.

Gonczi et al. (1992) and Froeschlé et al. (1993) studied a relationship between 96P and the Quadrantids, while also considering the Poynting-Robertson drag. Besides other facts, they found that the Quadrantids could only have been released from the comet nucleus 2000 years ago.

Before McIntosh's suggestion of the relationship between the comet 96P and Quadrantids, other objects were suggested as the parent of this stream. Hasegawa (1979) derived the orbital elements of several historical comets and concluded that comet C/1490 Y1 might be the parent comet of the Quadrantids. Williams \& Wu (1993) investigated this possibility and concluded that the Hasegawa's hypothesis is consistent with the data if the eccentricity of the comet is assumed to be 0.77 (instead approximating its orbit by parabola). They also showed that some dust grains ejected from C/1490 Y1 five millennia ago would have behaved like the Quadrantids. Williams \& Wu (see also Micheli et al. 2008) concluded that another historical comet, C/1385 U1, is probably identical to C/1490 Y1. Searching for other Quadrantid parents, Williams \& Collander-Brown (1998) suggested that, besides 96P, asteroid 5496 (1973 NA) could contribute to the stream.

The last period of the 96P-complex studies started after Jenniskens (2004) identified asteroid 2003 EH1 as the parent of the Quadrantids. Subsequently, Williams et al. (2004) summarized the situation with the Quadrantid parent to date, with special attention to $2003 \mathrm{EH} 1$. They revealed that around AD 1500 the orbit of the latter was very similar to the orbit of the Quadrantids. Furthermore, in 1491, the orbits of a few of the clones they considered were very similar to the orbit of C/1490 Y1, and their position on the sky also roughly matched that of the comet.

The relationship between asteroid 2003 EH1 and Quadrantids was further studied by Porubčan \& Kornoš (2005), as well as by Ryabova \& Nogami (2005). Wiegert \& Brown (2005) studied the complex especially considering that the Quadrantids had not been observed prior to 1835 , and several other objects are in orbits close to the orbital phase space of asteroid 2003 EH1. Specifically, they listed comets D/1783 W1 (Pigott), 5D/1846 D2 (Brorsen), D/1892 T1 (Barnard 3), 96P/1986 J2 (Machholz 1), and P/1994 P1 (Machholz 2); asteroids 1994 JX, 1999 LT1, 2000 PG3, 2002 AR129, 2002 KF4, 2002 UO3, 2003 YS1, and 2004 BZ74; as well as meteor showers Arietids and $\delta$-Aquarids S. Ki-Won et al. (2009) noticed a remarkable similarity of the orbits of all Quadrantids, comet C/1490 Y1, and asteroid $2003 \mathrm{EH} 1$.

Although a lot of information about the 96P meteoroid complex has been gained during past decades, it is still worthwhile investigating this complex. The present computational technique enables modeling the stream represented by many thousands of test particles, and the models can be created for several ejection moments in the past. The relatively large numbers of particles enable us to reveal both qualitative and some quantitative dynamical characteristics. In this article, we present a new, comprehensive study of the meteoroid complex of comet 96P/Machholz. Besides confirming the existing knowledge, we also reveal out some new structural aspects of the complex.

Before we start our description, we should clarify the terminology used here. Words "complex" and "stream" are synonyms referring to the whole structure of meteoroids released from the parent comet. The complex consists of several "filaments". If the particles of a filament hit the Earth, they can cause the meteor "shower" corresponding to the filament.

\section{Modeling of the stream dynamics}

To study the orbital evolution of test particles in the modeled, theoretical stream, we follow the procedure suggested by Neslušan (1999). The procedure, with some improvements, has recently been described in more detail in the paper by Tomko \& Neslušan (2010). Here, we briefly recall the individual steps.

(1) Integration of the motion of the given parent body backward in time for the period, which equals a suitably chosen number, $n$, of nominal orbital periods of parent body, $P_{\mathrm{o}}$. In more detail, the backward integration is stopped exactly at the time of the comet perihelion passage, which is closest to the chosen, above-mentioned period. We consider the gravitational perturbations of eight planets from Mercury to Neptune. Their initial position and velocity vectors are taken from the JPL ephemeris (ftp: //ssd. jpl .nasa.gov/pub/eph/ planets/bsp/; DE406). Integrator RA15, developed by Everhart (1985) within the MERCURY package (Chambers 1999), is used to run the numerical integration.

(2) Modeling of the theoretical stream in the moment of the perihelion passage of the parent body, which was reached at the previous step.

(3) Numerical integration of the stream particles from the moment of their assumed ejection (in step (2)) until the present. Integrator RA15 within the MERCURY package is again used. The final characteristics of eight perturbing planets and the parent body at the end of integration in step (1) are taken as initial in this step. Here, the standard output from integration is made every ten years.

(4) Analysis of main evolutionary features of the theoretical stream.

(5) Selection of the test particles that move in orbits passing in the vicinity of the Earth's orbit, at a distance shorter than $0.05 \mathrm{AU}$. These particles are regarded as the part of the stream colliding with the Earth and, thus, causing an eventual meteor shower.

(6) Analysis of the dynamical evolution of the Earth-orbit approaching part of the theoretical stream. If there are enough particles, the characteristics of an eventual meteor shower are predicted. For calculating the angular distance of the mean radiant from the Sun, $\gamma$ (see Table 1), the equatorial coordinates of the Sun are taken from the Astronomical 
Table 1. Mean geophysical characteristics of the predicted meteor showers.

\begin{tabular}{|c|c|c|c|c|c|c|c|c|c|}
\hline Shower & $t_{\mathrm{evol}}$ & $t_{\mathrm{act}} / \mathrm{D} . \mathrm{B}$. & $\left\langle\lambda_{\odot}\right\rangle$ & $\alpha_{\mathrm{g}}$ & $\delta_{\mathrm{g}}$ & $V_{\mathrm{g}}$ & $V_{\mathrm{h}}$ & $\gamma$ & $A / N_{\text {sel. }}$ \\
\hline \multirow[t]{8}{*}{$1-\mathrm{st}$} & 500 & Jun. 12-Jun. 16 & $83.177 \pm 0.737$ & $47.2 \pm 0.5$ & $+23.9 \pm 0.4$ & $40.4 \pm 1.1$ & $35.5 \pm 0.9$ & 32.3 & 0.0038 \\
\hline & 1000 & Jun. 4-Jun. 15 & $79.564 \pm 1.846$ & $45.8 \pm 1.3$ & $+23.0 \pm 0.6$ & $42.8 \pm 2.4$ & $38.4 \pm 1.6$ & 30.2 & 0.0916 \\
\hline & 2000 & Jun. 5-Jun. 15 & $82.218 \pm 1.778$ & $47.3 \pm 1.1$ & $+23.7 \pm 0.6$ & $42.7 \pm 2.0$ & $37.6 \pm 1.6$ & 31.3 & 0.0237 \\
\hline & 2900 & May 5-May 30 & $56.614 \pm 10.811$ & $31.2 \pm 8.1$ & $+16.6 \pm 0.6$ & $37.9 \pm 6.1$ & $38.8 \pm 2.3$ & 22.1 & 0.0005 \\
\hline & 3000 & Jun. 1 -Jun. 15 & $79.524 \pm 2.737$ & $45.5 \pm 2.0$ & $+23.4 \pm 0.9$ & $43.9 \pm 2.3$ & $39.1 \pm 1.5$ & 30.4 & 0.0059 \\
\hline & 3100 & May 17-Jun. 14 & $74.929 \pm 8.055$ & $42.5 \pm 5.1$ & $+23.3 \pm 1.0$ & $41.2 \pm 3.5$ & $38.1 \pm 1.6$ & 28.6 & 0.0055 \\
\hline & 3200 & Jun. 7-Jun. 15 & $81.719 \pm 1.236$ & $47.4 \pm 1.0$ & $+24.4 \pm 0.8$ & $43.1 \pm 2.2$ & $38.4 \pm 1.6$ & 30.7 & 0.0379 \\
\hline & 4000 & May 31-Jun. 17 & $81.013 \pm 4.270$ & $46.6 \pm 3.8$ & $+24.6 \pm 1.1$ & $42.7 \pm 2.0$ & $38.2 \pm 1.5$ & 30.7 & 0.0332 \\
\hline \multirow[t]{10}{*}{ 2-nd } & 500 & & - & - & - & - & - & - & 0.0000 \\
\hline & 1000 & & - & - & - & - & - & - & 0.0000 \\
\hline & 2000 & & - & - & - & - & - & - & 0.0000 \\
\hline & 2900 & Jul. 13-Aug. 4 & $120.541 \pm 4.714$ & $332.4 \pm 3.0$ & $-7.1 \pm 1.9$ & $44.3 \pm 3.1$ & $38.6 \pm 2.1$ & 148.5 & 0.0035 \\
\hline & 3000 & Jul. 16-Aug. 7 & $124.036 \pm 15.104$ & $333.3 \pm 3.5$ & $-7.5 \pm 0.8$ & $39.0 \pm 2.8$ & $36.0 \pm 4.0$ & 151.3 & 0.0002 \\
\hline & 3100 & Jul. 13-Jul. 16 & $112.108 \pm 1.549$ & $328.5 \pm 0.5$ & $-8.3 \pm 0.2$ & $43.9 \pm 0.5$ & $36.1 \pm 0.9$ & 144.1 & 0.0002 \\
\hline & 3200 & & - & - & - & - & - & - & 0.0000 \\
\hline & 4000 & Aug. 4.6 & 132.407 & 343.1 & -3.2 & 44.0 & 38.7 & 149.0 & 0.0001 \\
\hline & $\mathrm{F}$ & Jul. 30-Aug. 16 & $136.222 \pm 4.770$ & $342.9 \pm 3.5$ & $-0.4 \pm 2.8$ & $39.6 \pm 2.5$ & $37.0 \pm 2.0$ & & 18 \\
\hline & $\mathrm{V}$ & Aug. 4-Aug. 20 & $139.349 \pm 3.351$ & $345.9 \pm 2.4$ & $+1.3 \pm 1.6$ & $39.1 \pm 1.6$ & $36.6 \pm 1.5$ & & 39 \\
\hline \multirow[t]{10}{*}{ 3-rd } & 500 & & - & - & - & - & - & - & 0.0000 \\
\hline & 1000 & & - & - & - & - & - & - & 0.0000 \\
\hline & 2000 & Jan. 2-Jan. 4 & $282.257 \pm 0.513$ & $227.1 \pm 2.4$ & $+50.2 \pm 1.0$ & $41.7 \pm 0.2$ & $38.95 \pm 0.07$ & 86.8 & 0.0010 \\
\hline & 2900 & Dec. 28-Jan. 6 & $281.575 \pm 1.392$ & $224.5 \pm 3.7$ & $+50.3 \pm 2.2$ & $42.4 \pm 0.5$ & $39.0 \pm 0.1$ & 88.7 & 0.0274 \\
\hline & 3000 & Jan. 1-Jan. 5 & $282.675 \pm 1.109$ & $226.4 \pm 3.5$ & $+49.4 \pm 1.3$ & $42.3 \pm 0.5$ & $38.9 \pm 0.1$ & 87.0 & 0.0056 \\
\hline & 3100 & Dec. $31-J a n .5$ & $282.253 \pm 1.213$ & $226.6 \pm 3.3$ & $+49.2 \pm 1.5$ & $42.4 \pm 0.5$ & $39.0 \pm 0.1$ & 86.5 & 0.0026 \\
\hline & 3200 & Dec. 30-Jan. 6 & $282.455 \pm 1.311$ & $227.6 \pm 3.4$ & $+48.8 \pm 1.0$ & $42.4 \pm 0.7$ & $39.0 \pm 0.1$ & 85.8 & 0.0023 \\
\hline & 4000 & Jan. 3.4 & 282.233 & 228.2 & +48.3 & 42.5 & 39.0 & 85.7 & 0.0001 \\
\hline & $\mathrm{F}$ & Jan. 2-Jan. 8 & $283.332 \pm 0.767$ & $230.4 \pm 2.2$ & $+49.2 \pm 1.0$ & $41.0 \pm 1.0$ & $38.8 \pm 0.6$ & & 59 \\
\hline & V & Dec. 24-Jan. 13 & $283.135 \pm 1.498$ & $229.8 \pm 3.2$ & $+49.6 \pm 1.7$ & $40.1 \pm 1.3$ & $38.1 \pm 0.8$ & & 804 \\
\hline \multirow[t]{8}{*}{ 4-th } & 500 & & - & - & - & - & - & - & 0.0000 \\
\hline & 1000 & & - & - & - & - & - & - & 0.0000 \\
\hline & 2000 & & - & - & - & - & - & - & 0.0000 \\
\hline & 2900 & Jun. 6-Jun. 27 & $86.270 \pm 4.122$ & $53.5 \pm 2.6$ & $+15.0 \pm 1.3$ & $44.1 \pm 2.7$ & $38.3 \pm 1.7$ & 31.7 & 0.0042 \\
\hline & 3000 & Jun. 19-Jun. 23 & $89.638 \pm 2.441$ & $56.4 \pm 1.5$ & $+16.1 \pm 0.8$ & $46.0 \pm 0.7$ & $39.57 \pm 0.03$ & 32.0 & 0.0002 \\
\hline & 3100 & Jun. 23.8 & 92.335 & 56.5 & +16.2 & 44.7 & 37.3 & 34.6 & 0.0001 \\
\hline & 3200 & & - & - & - & - & - & - & 0.0000 \\
\hline & 4000 & Jun. 19.6 & 88.563 & 56.5 & +14.4 & 44.3 & 38.7 & 31.4 & 0.0001 \\
\hline \multirow[t]{10}{*}{ 5-th } & 500 & & - & - & - & - & - & - & 0.0000 \\
\hline & 1000 & Jul. 18-Jul. 31 & $120.806 \pm 2.468$ & $335.3 \pm 1.6$ & $-15.7 \pm 0.8$ & $43.2 \pm 2.4$ & $38.1 \pm 1.9$ & 149.0 & 0.0682 \\
\hline & 2000 & Jul. 20-Aug. 19 & $125.804 \pm 4.072$ & $338.4 \pm 2.6$ & $-15.3 \pm 0.8$ & $41.8 \pm 2.8$ & $38.1 \pm 1.6$ & 150.9 & 0.0171 \\
\hline & 2900 & Jul. 16-Sep. 17 & $143.128 \pm 19.025$ & $347.7 \pm 12.7$ & $-13.7 \pm 3.3$ & $36.1 \pm 3.9$ & $38.2 \pm 1.3$ & 158.4 & 0.0010 \\
\hline & 3000 & Jul. 22-Aug. 6 & $126.371 \pm 3.562$ & $339.3 \pm 2.3$ & $-16.2 \pm 0.7$ & $41.2 \pm 2.3$ & $37.8 \pm 1.4$ & 150.8 & 0.0149 \\
\hline & 3100 & Jul. 20-Aug. 17 & $127.348 \pm 4.326$ & $339.8 \pm 2.7$ & $-16.0 \pm 0.9$ & $41.1 \pm 2.7$ & $38.0 \pm 1.5$ & 151.2 & 0.0162 \\
\hline & 3200 & Jul. 16-Aug. 8 & $124.753 \pm 2.965$ & $338.3 \pm 1.9$ & $-16.3 \pm 0.7$ & $42.0 \pm 2.5$ & $38.0 \pm 1.6$ & 150.2 & 0.0221 \\
\hline & 4000 & Jul. 16-Aug. 6 & $125.307 \pm 4.315$ & $338.7 \pm 3.6$ & $-16.5 \pm 1.3$ & $42.0 \pm 2.3$ & $38.2 \pm 1.5$ & 150.4 & 0.0236 \\
\hline & $\mathrm{F}$ & Jul. 25-Aug. 16 & $131.989 \pm 5.217$ & $343.4 \pm 4.2$ & $-15.1 \pm 1.1$ & $40.0 \pm 1.6$ & $37.7 \pm 1.2$ & & 36 \\
\hline & $\mathrm{V}$ & Jul. 22-Aug. 20 & $129.576 \pm 5.435$ & $342.4 \pm 4.7$ & $-16.0 \pm 1.6$ & $39.5 \pm 2.1$ & $36.8 \pm 1.7$ & & 452 \\
\hline \multirow[t]{8}{*}{ 6-th } & 500 & & - & - & - & - & - & - & 0.0000 \\
\hline & 1000 & & - & - & - & - & - & - & 0.0000 \\
\hline & 2000 & Dec. $18-$ Dec. 20 & $267.363 \pm 1.587$ & $87.9 \pm 9.4$ & $-41.9 \pm 5.1$ & $26.1 \pm 3.0$ & $40.2 \pm 0.2$ & 115.5 & 0.0002 \\
\hline & 2900 & Dec. 19-Jan. 3 & $274.499 \pm 7.852$ & $139.0 \pm 11.7$ & $-53.6 \pm 4.5$ & $41.6 \pm 2.7$ & $39.3 \pm 0.1$ & 94.8 & 0.0004 \\
\hline & 3000 & Dec. $22-$ Dec. 24 & $271.161 \pm 1.072$ & $122.1 \pm 8.3$ & $-51.3 \pm 0.9$ & $37.2 \pm 2.6$ & $39.5 \pm 0.2$ & 101.3 & 0.0003 \\
\hline & 3100 & Dec. 23.3 & 271.488 & 126.0 & -51.3 & 38.8 & 39.6 & 100.2 & 0.0001 \\
\hline & 3200 & Dec. 16-Dec. 18 & $265.175 \pm 1.457$ & $93.7 \pm 8.1$ & $-44.4 \pm 3.3$ & $28.6 \pm 2.6$ & $40.1 \pm 0.1$ & 112.4 & 0.0002 \\
\hline & 4000 & Dec. 24-Jan. 5 & $277.845 \pm 1.857$ & $153.6 \pm 8.0$ & $-53.6 \pm 2.7$ & $45.8 \pm 1.2$ & $39.3 \pm 0.1$ & 90.2 & 0.0262 \\
\hline
\end{tabular}

Notes. $t_{\text {evol }}$ - period of the following of orbital evolution (the theoretical stream was modeled before this time); D.B. - database from which the observed counterpart of filament is selected ( $\mathrm{F}$ - photographic, $\mathrm{V}$ - video-meteor); $t_{\text {act }}$ - typical period of the activity of shower during a year; $\left\langle\lambda_{\odot}\right\rangle$ - mean ecliptical longitude of the Sun at the moment of collision of shower particles with the Earth; $\alpha_{\mathrm{g}}$ and $\delta_{\mathrm{g}}-$ equatorial coordinates of geocentric radiant; $V_{\mathrm{g}}$ and $V_{\mathrm{h}}$ - geocentric and heliocentric velocity; $\gamma$ - angular distance of the mean radiant from the Sun in time corresponding to the mean longitude; $A$ - amount of the particles of whole modeled stream constituting the given shower; $N_{\text {sel. }}-$ the number of real meteors selected from the given database. Time $t_{\mathrm{evol}}$ is given in years, the angular quantities are given in degrees, and velocities in $\mathrm{km} \mathrm{s}^{-1}$. 
Almanac for Years 2004, 2005, 2006, and 2008 (published in 2002, 2003, 2004, and 2006, respectively).

(7) Identification of the Earth-orbit approaching particles with the actually observed meteors. The photographic (Lindblad et al. 2003) and radio-meteor (Hawkins 1963; Sekanina \& Southworth 1975; Lindblad 2003, priv. comm.) IAU MDC databases, as well as the SonotaCo video-meteor database (SonotaCo 2009) are used in this identification. The first, second, and third catalogs contain the data on the 4581, 62906 , and 64650 meteors, respectively. The proper identification is done using the "break-point" method suggested by Neslušan et al. (1995, 2012). In this work, we deal with more or less compact showers, therefore we do not modify the original break-point method (as suggested in Tomko \& Neslušan 2010), but we calculate the $D$-discriminant between the mean orbit of a predicted shower and every orbit of a real meteor.

\section{Past evolution of $96 \mathrm{P}$ orbit}

The initial orbit of every modeled theoretical particle depends on the position of the parent, comet 96P, in the moment of assumed ejection. Owing to a determination uncertainty, as well as the action of stochastic nongravitational effects, the orbit in the time of the ejection is, however, not known exactly.

The nominal orbit of $96 \mathrm{P}$ is the best fit for the available observations of this comet. Its real orbit can be slightly different. The optimal ways have been suggested to construct the clones of the nominal orbit which fulfill the probable orbital phase space in which the real orbit can be situated (Chernitsov et al. 1998; Milani 1999; Milani et al. 2005). Since the nominal orbit of $96 \mathrm{P}$ is known with the uncertainty of its elements and since we only need to constrain the possible six dimensional orbital phase space, in which the real orbit can be situated, we proceed in a simpler way to construct the clones. We construct a "shell" of alternative orbits bordering the relevant phase space. The real orbit of $96 \mathrm{P}$ can be situated within this shell with a probability at the $1-\sigma$ level. Utilizing the circumstance that each element of 96P's nominal orbit is determined with its standard deviation, $\sigma$, we construct the "grid" of cloned orbits by considering three values of each element: the nominal value $X$, the nominal value reduced by the determination error $X-\sigma_{X}$, and the nominal value increased by $\sigma_{X}$, i.e. $X+\sigma_{X}$. The grid consists of the cloned orbits, which are combinations of all three values of each of six elements (i.e. $3^{6}=729$ orbits). Integrating these clones backward in time for five millennia, we delimit an uncertainty corridor of the real orbit.

The behavior of the orbital elements for the nominal orbit of $96 \mathrm{P}$ is plotted with the red line in each plot in Fig. 1. The behavior of the elements of clones is also plotted in this figure with the green line. A significant difference between the nominal orbit and orbits of clones appears at about 4100 years before the present.

In a similar way, we map the uncertainty of $96 \mathrm{P}$ orbit due to the possible nongravitational effects. For a short period covering four apparitions of 96P (1986-2002), Sekanina \& Chodas (2005) found non-gravitational parameters $A_{1}=(+0.0264 \pm$ $0.0063) \times 10^{-8} \mathrm{AU} \mathrm{day}^{-2}, A_{2}=(-0.0001500 \pm 0.0000037) \times$ $10^{-8} \mathrm{AU} \mathrm{day}^{-2}$. On a larger timescale, the parameters change significantly, and their values are not known. We cannot exclude that the magnitude of the parameters was much larger in the past. For several millennia of the investigation of 96P orbital evolution, we therefore determine the typical positive and negative statistical values based on the data given in the Catalogue of Cometary Orbits, Sixth Edition (Marsden 1989).

Considering parameters $A_{1}$ and $A_{2}$ listed in the catalog for a set of periodic comets, we find the mean negative value of parameter $A_{1}$ equal to $A_{1-}=-0.412570 \pm 0.447186$ and mean positive value of $A_{1}$ equal to $A_{1+}=0.863065 \pm 2.012802$. The analogous values for parameter $A_{2}$ are $A_{2-}=-0.102083 \pm 0.073139$ and $A_{2+}=0.072534 \pm 0.021792$. Parameter $A_{3}$ is usually very small. That is why we consider $A_{3}=0$. With the mean values with their standard deviations, $\sigma$, we again construct the clones of nominal orbit using all combinations of seven values, $A_{-}-\sigma_{A_{-}}, A_{-}, A_{-} / 2,0, A_{+} / 2, A_{+}$, and $A_{+}+\sigma_{A_{+}}$of both $A_{1}$ and $A_{2}$ $\left(7^{2}=49\right.$ orbits, in total).

The numerical integration of these clones backward in time reveals that the uncertainty due to the possible nongravitational force appears to be much smaller than the one caused by a determination error. In the resolution of Fig. 1, no deviation of any element from the nominal value can be distinguished.

It seems that the resonance action of Jupiter tends to sweep out some eventual deviations from a relatively narrow phase space in which the orbit of $96 \mathrm{P}$ is situated. The modeling of $96 \mathrm{P}$ stream is thus not significantly influenced by the uncertainty of the orbit of this parent for a long period in the past.

In Fig. 2, we show the evolution of the minimum distance between the orbit of $96 \mathrm{P}$ and the orbit of the Earth. We see that the comet passed the Earth's orbit closely (within $0.1 \mathrm{AU}$ ) in several short periods. For the rest, the orbits of $96 \mathrm{P}$ and the Earth were quite distant.

\section{Modeled streams}

A meteoroid-stream parent body ejects the meteoroids from its surface along a shorter or longer arc of its orbit around the perihelion. The studied comet could have been supplying the particles to the current stream repeatedly, during a period covering a few thousand perihelion passages. A complete modeling of the whole process of stream formation and evolution would therefore require considering and numerically integrating the orbits of a huge number of particles. Such a demand would scarcely be satisfied with the help of supercomputers working with the currently best performance and certainly exceeds the performance of the computational technique available to our meteoroid-stream study, so we mapped the evolution by modeling the stream only at the perihelion point and only for several chosen moments of perihelion passages in the past.

Specifically, we modeled the streams in times corresponding to 96P's perihelion passages about 500, 1000, 2000, 2900, 3000, 3100,3200 , and 4000 years ago. Hereinafter, we refer to these models as, e.g., MT500 (modeling for a time before 500 years). Times 500, 1000, 2000, 3000, and 4000 years are chosen ad hoc to cover the entire expected interval for the evolution of a prevaling part of the meteoroids in the current stream. Modeling of a long time before 4000 is not very reasonable (see Sect. 3). To see some specific aspects of the evolution on the timescale of a century, we also modeled the stream at times 2900 (3000 is also used in this context), 3100, and 3200 years before the present.

The orbital evolution of a prevailing part of the considered test particles has the same features as the evolution of 96P's orbit itself, except for the phase that can be shifted about the entire cycle of the quasi-periodic variation. One circulation period of the longitude of ascending node and, at the same time, argument of perihelion lasts 8.2 millennia. (The period of libration of all perihelion distance, eccentricity, and inclination is a half of the period of $\Omega$-circulation. This is obviously why 
L. Neslušan et al.: The meteor-shower complex of 96P/Machholz revisited
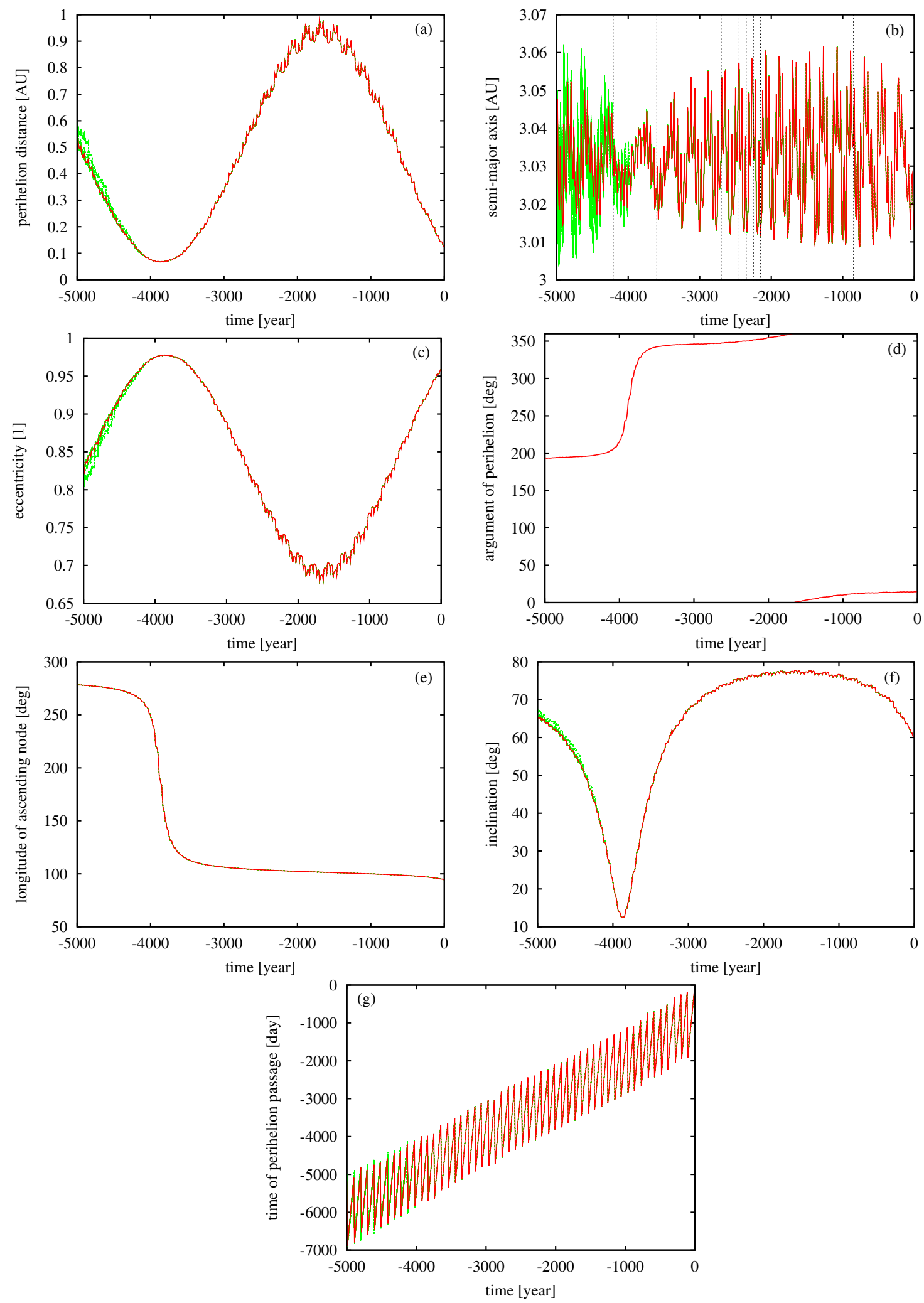

Fig. 1. Uncertainty of perihelion distance a), semi-major axis b), eccentricity c), argument of perihelion d), longitude of asceding node e), inclination to the ecliptic f), and time of perihelion passage $\mathbf{g}$ ) when reconstructing the orbit of comet 96P backward for 5 millennia. The backward evolution of any given element is shown with the help of cloned orbits differring by 1- $\sigma$ from the nominal value (see the text of Sect. 3).

the authors often speak about the orbit-evolution cycle lasting $\sim 4$ millennia.) It is well-known (Babadzhanov \& Obrubov 1992) that the nodes of 96P's orbit cross the Earth's orbit at eight points during the comet-orbit evolution cycle. The evolution of the cross-point position for one period of the $\Omega$-circulation is illustrated in Fig. 3. The solid red curve illustrates the shift in the descending node (the radiant of corresponding meteors is situated northward of the ecliptic) and dashed blue curve the shift in the ascending node (the radiant is southward to the ecliptic) of the comet orbit. It is seen that each node basically crosses the 


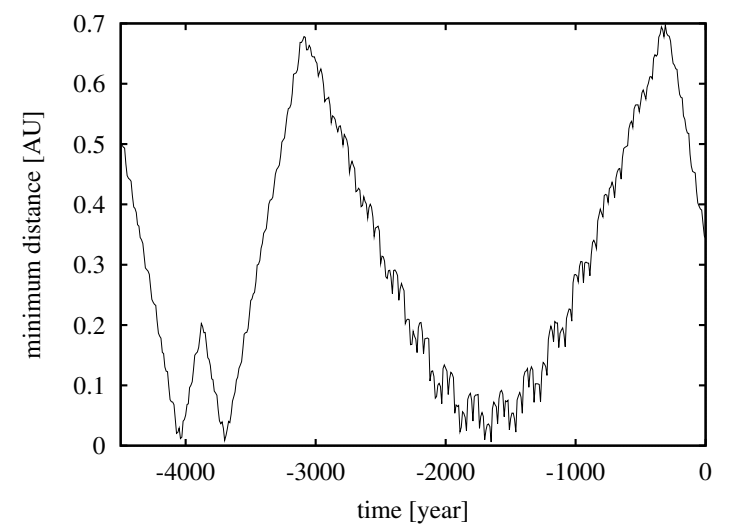

Fig. 2. Evolution of the minimum distance between the orbits of comet 96P/Machholz and Earth from time 4500 years before the present to the present.

Earth's orbit four times, twice at the outward and twice at the inward shift. (More exactly, the narrow loop of the dashed blue curve does not reach Earth's orbit when considering the nominal orbit of 96P. However, the corresponding loop of a lot of meteoroids approaching the Earth's orbit does so. Therefore, we also suppose two crossing points for this loop.)

The above-mentiond narrow loop related to the descending node (solid red curve in Fig. 3) corresponds to the well-known meteor shower Quadrantids (see a more detailed discussion in Sect. 4.3). Although Babadzhanov \& Obrubov assigned the crosspoint of the outward shifting part of the curve to the Quadrantids and the inward shifting part to another shower, Ursids, we find that this conceptual resolution is impossible in a quantitative study of a real or a theoretical Quadrantid stream. For a large number of meteoroids, the interval of the crosspoints related to the nodes that shift outward is almost entirely overlapped by the interval of those crosspoints that are related to the nodes that shift inward. The orbital parameters and radiants corresponding to meteoroids in these intervals also lie in the appropriate overlapping intervals. One has no serious quantitative criterion for distinguishing two showers. The same conclusion can be drawn for the narrow loop related to the ascending node (dashed blue curve in Fig. 3), so we assign both crosssections of the given narrow loop only to a single northern and single southern meteor shower (Sects. 4.3 and 4.6). Thus, we predict that the complex of $96 \mathrm{P}$ causes six meteor showers observable in the Earth's atmosphere.

We divide that part of each modeled stream that is approaching the Earth into the filaments on the basis of the radiant position of meteors. The members of a given filament have a common radiant area. In detail, the radiant areas of filaments 1, 2, and 3 are situated northward of the ecliptic (northern branches of corresponding showers) and those of filaments 4, 5 , and 6 southward of the ecliptic (southern shower branches). Furthermore, the radiant area of filament 1 is constrained with geocentric-radiant coordinates $18^{\circ}<\alpha_{\mathrm{g}}<55^{\circ}$, filament 2 with $320^{\circ}<\alpha_{\mathrm{g}}<350^{\circ}$, filament 3 with $210^{\circ}<\alpha_{\mathrm{g}}<280^{\circ}$ and $0^{\circ}<\delta_{\mathrm{g}}<65^{\circ}$, filament 4 with $30^{\circ}<\alpha_{\mathrm{g}}<65^{\circ}$, filament 5 with $\alpha_{\mathrm{g}}<17^{\circ}$ or $\alpha_{\mathrm{g}}>320^{\circ}$, and filament 6 with $80^{\circ}<\alpha_{\mathrm{g}}<170^{\circ}$ and $-60^{\circ}<\delta_{\mathrm{g}}<-38^{\circ}$. The other parameters could, of course, also be used to divide the part of each modeled stream that is approaching the Earth into the individual filaments.

The obtained mean geophysical characteristics of all six filaments are given in Table 1 and mean orbital elements in Table 2. In this table, we can see, e.g., that mean values of argument of

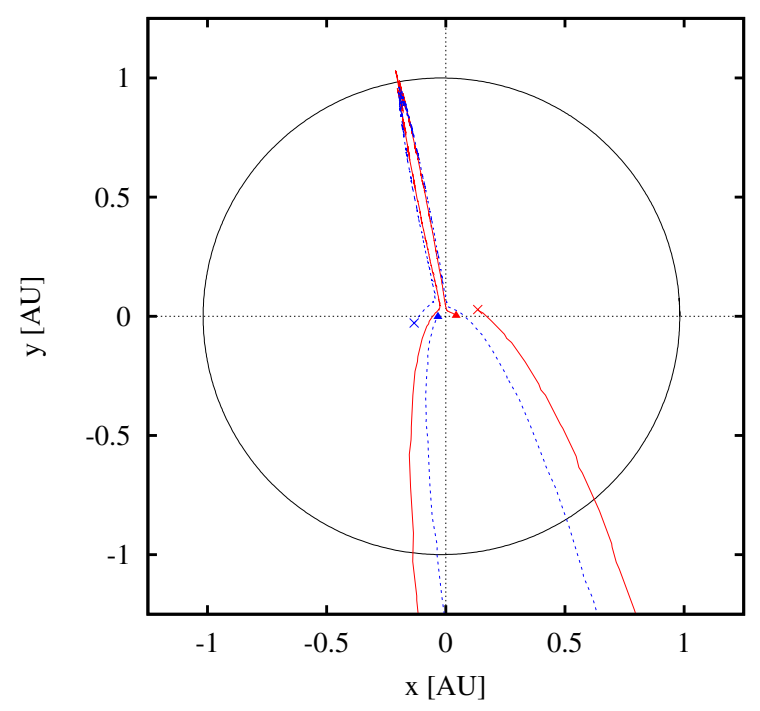

Fig. 3. Evolution of the orbital node position of comet 96P/Machholz during one circulation period of the longitude of ascending node (which is the same as the circulation period of argument of perihelion). The solid red (dashed blue) curve shows the shift in the descending (ascending) node corresponding to the radiant northward (southward) of the ecliptic. The crosses indicate the starting positions and full triangles the final positions of the nodes in the considered circulation period.

perihelion for individual filaments differ each other quite well (except for filaments 1 and 6, which, however, clearly differ in perihelion distance).

The distributions of orbital elements of the Earth-orbit approaching particles are shown in Fig. 4. Two high peaks can be seen in almost all element distributions. These peaks mostly correspond to the two most numerous filaments of the entire stream, which we label, hereinafter, as filaments 1 and 5. We say "mostly" because the values of given element of particles forming two filaments are often spread in neighboring, partially overlapping intervals with a common, single peak. For example, the left wing of the distribution of argument of perihelion (Fig. 4d), $\omega$, in filament 3 (mean theoretical values of $\omega$ range from $172.8^{\circ}$ to $178.2^{\circ}$, see Table 2 ) obviously coincides with the right wing of $\omega$-distribution in filament 5 (in which the mean theoretical values range from $136.6^{\circ}$ to $157.7^{\circ}$ ).

The complex structure of the stream is moreover illustrated with the predicted radiants of particles expected to collide with the Earth in the present. The positions of the radiants are plotted in Fig. 5.

The number of particles in a given filament varies from model to model. Also the mean characteristics of the filaments with fewer than five members in a model are given in Tables 1 and 2 , because they are significant for some other modeling time(s).

Despite a relatively large distance for the orbit of $96 \mathrm{P}$ from the Earth's orbit (Fig. 2) in the moments of several modelings of its stream, our results confirm that the comet could feed enough with the particles also those filaments of the stream which were deflected far from the comet's orbit and approached the orbit of the Earth.

Among the modeled streams, stream MT2900 is peculiar because filaments 2,3 , and 4 are much more abundant in this model than they are in the other models. In contrast, filaments 1 and 5 are relatively depleted in the MT2900 model. It seems that the supply of the particles to the individual filaments alternate: mostly, the particles are supplied to the most abundant 
Table 2. Mean orbital characteristics with the dispersion (characterized by standard deviation) of the predicted meteor showers associated with the parent body considered.

\begin{tabular}{|c|c|c|c|c|c|c|c|}
\hline Filament & $t_{\mathrm{evol}} / \mathrm{D} . \mathrm{B}$. & $q$ & $a$ & $e$ & $\omega$ & $\Omega$ & $i$ \\
\hline \multirow[t]{9}{*}{1 -st } & 500 & $0.043 \pm 0.003$ & $3.011 \pm 0.063$ & $0.986 \pm 0.001$ & $18.1 \pm 1.3$ & $86.1 \pm 1.3$ & $33.7 \pm 2.5$ \\
\hline & 1000 & $0.053 \pm 0.008$ & $3.091 \pm 0.046$ & $0.983 \pm 0.003$ & $24.9 \pm 3.2$ & $78.9 \pm 3.4$ & $25.2 \pm 3.2$ \\
\hline & 2000 & $0.044 \pm 0.006$ & $3.042 \pm 0.028$ & $0.985 \pm 0.002$ & $21.5 \pm 3.1$ & $82.5 \pm 3.5$ & $30.3 \pm 4.3$ \\
\hline & 2900 & $0.151 \pm 0.076$ & $3.654 \pm 0.312$ & $0.960 \pm 0.018$ & $37.0 \pm 6.9$ & $60.9 \pm 6.0$ & $9.7 \pm 5.6$ \\
\hline & 3000 & $0.054 \pm 0.012$ & $3.043 \pm 0.036$ & $0.982 \pm 0.004$ & $26.6 \pm 3.3$ & $77.4 \pm 3.8$ & $26.5 \pm 3.9$ \\
\hline & 3100 & $0.079 \pm 0.042$ & $3.145 \pm 0.185$ & $0.976 \pm 0.011$ & $28.7 \pm 6.8$ & $75.1 \pm 7.4$ & $26.3 \pm 5.0$ \\
\hline & 3200 & $0.053 \pm 0.007$ & $2.986 \pm 0.032$ & $0.982 \pm 0.002$ & $25.0 \pm 2.6$ & $80.9 \pm 3.0$ & $29.6 \pm 3.3$ \\
\hline & 4000 & $0.056 \pm 0.009$ & $3.053 \pm 0.112$ & $0.982 \pm 0.003$ & $25.0 \pm 3.0$ & $80.7 \pm 5.3$ & $31.6 \pm 3.7$ \\
\hline & $\mathrm{R}$ & $0.082 \pm 0.017$ & $2.2 \pm 2.3$ & $0.948 \pm 0.024$ & $27.2 \pm 3.8$ & $77.8 \pm 3.0$ & $26.4 \pm 2.7$ \\
\hline \multirow{11}{*}{ 2-nd } & 500 & - & - & - & - & - & - \\
\hline & 1000 & - & - & - & - & - & - \\
\hline & 2000 & - & - & - & - & - & - \\
\hline & 2900 & $0.035 \pm 0.016$ & $2.931 \pm 0.082$ & $0.988 \pm 0.005$ & $339.8 \pm 5.2$ & $121.8 \pm 5.5$ & $23.4 \pm 3.9$ \\
\hline & 3000 & $0.09 \pm 0.11$ & $3.25 \pm 0.68$ & $0.974 \pm 0.027$ & $348.7 \pm 1.6$ & $106.5 \pm 6.0$ & $25 \pm 29$ \\
\hline & 3100 & $0.014 \pm 0.001$ & $2.751 \pm 0.020$ & $0.9949 \pm 0.0005$ & $349.2 \pm 1.2$ & $111.2 \pm 1.9$ & $45.5 \pm 4.4$ \\
\hline & 3200 & - & - & - & - & - & - \\
\hline & 4000 & 0.033 & 3.271 & 0.990 & 340.4 & 132.8 & 21.8 \\
\hline & $\mathrm{F}$ & $0.084 \pm 0.024$ & $2.628 \pm 0.779$ & $0.963 \pm 0.023$ & $330.6 \pm 4.2$ & $136.2 \pm 4.8$ & $21.3 \pm 3.2$ \\
\hline & $\mathrm{V}$ & $0.086 \pm 0.016$ & $2.269 \pm 0.454$ & $0.960 \pm 0.012$ & $330.5 \pm 3.2$ & $139.3 \pm 3.4$ & $22.5 \pm 2.3$ \\
\hline & $\mathrm{R}$ & $0.111 \pm 0.039$ & $1.7 \pm 1.3$ & $0.919 \pm 0.042$ & $330.4 \pm 7.4$ & $130.1 \pm 8.5$ & $24.4 \pm 4.9$ \\
\hline \multirow{11}{*}{3 -rd } & 500 & - & - & - & - & - & - \\
\hline & 1000 & - & - & - & - & - & - \\
\hline & 2000 & $0.976 \pm 0.019$ & $3.074 \pm 0.035$ & $0.683 \pm 0.007$ & $175.7 \pm 4.5$ & $282.3 \pm 0.5$ & $72.9 \pm 0.6$ \\
\hline & 2900 & $0.977 \pm 0.028$ & $3.086 \pm 0.044$ & $0.683 \pm 0.009$ & $178.2 \pm 7.0$ & $281.6 \pm 1.4$ & $74.3 \pm 1.2$ \\
\hline & 3000 & $0.976 \pm 0.028$ & $3.073 \pm 0.043$ & $0.682 \pm 0.010$ & $175.5 \pm 5.8$ & $282.7 \pm 1.1$ & $74.2 \pm 1.0$ \\
\hline & 3100 & $0.974 \pm 0.029$ & $3.078 \pm 0.047$ & $0.683 \pm 0.012$ & $174.6 \pm 6.0$ & $282.2 \pm 1.2$ & $74.3 \pm 1.2$ \\
\hline & 3200 & $0.965 \pm 0.026$ & $3.076 \pm 0.079$ & $0.686 \pm 0.011$ & $172.8 \pm 5.3$ & $282.4 \pm 1.3$ & $74.2 \pm 1.7$ \\
\hline & 4000 & 0.960 & 3.079 & 0.688 & 170.7 & 282.2 & 74.5 \\
\hline & $\mathrm{F}$ & $0.978 \pm 0.004$ & $3.008 \pm 0.508$ & $0.667 \pm 0.050$ & $170.8 \pm 3.5$ & $283.3 \pm 0.8$ & $71.7 \pm 1.6$ \\
\hline & V & $0.978 \pm 0.005$ & $2.610 \pm 0.498$ & $0.613 \pm 0.066$ & $171.8 \pm 5.5$ & $283.1 \pm 1.5$ & $70.6 \pm 2.3$ \\
\hline & $\mathrm{R}$ & $0.969 \pm 0.014$ & $3.217 \pm 1.020$ & $0.673 \pm 0.087$ & $167.1 \pm 8.2$ & $282.8 \pm 2.5$ & $70.8 \pm 3.5$ \\
\hline \multirow[t]{9}{*}{ 4-th } & 500 & - & - & - & - & - & - \\
\hline & 1000 & - & - & - & - & - & - \\
\hline & 2000 & - & - & - & - & - & - \\
\hline & 2900 & $0.033 \pm 0.015$ & $2.902 \pm 0.078$ & $0.989 \pm 0.005$ & $199.0 \pm 4.1$ & $265.7 \pm 4.0$ & $25.1 \pm 4.0$ \\
\hline & 3000 & $0.025 \pm 0.009$ & $2.824 \pm 0.073$ & $0.991 \pm 0.003$ & $198.9 \pm 3.1$ & $267.3 \pm 2.7$ & $24.3 \pm 1.1$ \\
\hline & 3100 & 0.014 & 2.753 & 0.995 & 191.8 & 272.7 & 36.0 \\
\hline & 3200 & - & - & - & - & - & - \\
\hline & 4000 & 0.036 & 3.302 & 0.989 & 200.3 & 268.2 & 31.1 \\
\hline & $\mathrm{R}$ & $0.067 \pm 0.023$ & $1.364 \pm 0.465$ & $0.945 \pm 0.028$ & $202.2 \pm 4.6$ & $264.2 \pm 4.0$ & $33.4 \pm 3.6$ \\
\hline \multirow[t]{11}{*}{5 -th } & 500 & - & - & - & - & - & - \\
\hline & 1000 & $0.043 \pm 0.008$ & $3.013 \pm 0.058$ & $0.986 \pm 0.002$ & $157.7 \pm 3.9$ & $301.4 \pm 4.5$ & $26.2 \pm 4.6$ \\
\hline & 2000 & $0.066 \pm 0.020$ & $3.102 \pm 0.075$ & $0.979 \pm 0.006$ & $153.1 \pm 4.0$ & $306.0 \pm 4.4$ & $24.0 \pm 3.6$ \\
\hline & 2900 & $0.188 \pm 0.104$ & $3.608 \pm 0.338$ & $0.950 \pm 0.024$ & $136.6 \pm 15.8$ & $320.5 \pm 19.4$ & $18.2 \pm 4.2$ \\
\hline & 3000 & $0.072 \pm 0.018$ & $3.049 \pm 0.069$ & $0.977 \pm 0.006$ & $152.4 \pm 4.2$ & $306.0 \pm 4.4$ & $27.6 \pm 3.6$ \\
\hline & 3100 & $0.078 \pm 0.022$ & $3.093 \pm 0.092$ & $0.975 \pm 0.006$ & $151.0 \pm 4.7$ & $307.2 \pm 5.1$ & $26.3 \pm 3.8$ \\
\hline & 3200 & $0.063 \pm 0.015$ & $3.038 \pm 0.085$ & $0.979 \pm 0.005$ & $153.6 \pm 3.6$ & $304.9 \pm 3.9$ & $28.2 \pm 3.8$ \\
\hline & 4000 & $0.068 \pm 0.015$ & $3.054 \pm 0.128$ & $0.978 \pm 0.005$ & $152.4 \pm 3.5$ & $305.6 \pm 4.9$ & $28.6 \pm 3.6$ \\
\hline & $\mathrm{F}$ & $0.093 \pm 0.022$ & $3.0 \pm 1.1$ & $0.966 \pm 0.013$ & $148.2 \pm 4.0$ & $312.0 \pm 5.2$ & $24.7 \pm 3.2$ \\
\hline & $\mathrm{V}$ & $0.090 \pm 0.026$ & $2.7 \pm 4.2$ & $0.960 \pm 0.020$ & $149.7 \pm 4.4$ & $309.6 \pm 5.4$ & $26.3 \pm 4.0$ \\
\hline & $\mathrm{R}$ & $0.080 \pm 0.031$ & $1.98 \pm 1.14$ & $0.949 \pm 0.032$ & $153.5 \pm 5.8$ & $306.9 \pm 5.8$ & $28.0 \pm 5.3$ \\
\hline \multirow[t]{8}{*}{ 6-th } & 500 & - & - & - & - & - & - \\
\hline & 1000 & - & - & - & - & - & - \\
\hline & 2000 & $0.897 \pm 0.007$ & $4.735 \pm 0.348$ & $0.810 \pm 0.012$ & $34.8 \pm 3.6$ & $87.5 \pm 2.2$ & $37.9 \pm 6.8$ \\
\hline & 2900 & $0.939 \pm 0.019$ & $3.285 \pm 0.177$ & $0.714 \pm 0.015$ & $10 \pm 12$ & $94.5 \pm 7.9$ & $72.2 \pm 5.9$ \\
\hline & 3000 & $0.958 \pm 0.027$ & $3.719 \pm 0.249$ & $0.741 \pm 0.020$ & $23.2 \pm 4.0$ & $91.1 \pm 1.1$ & $62.4 \pm 5.7$ \\
\hline & 3100 & 0.910 & 3.581 & 0.746 & 21.6 & 91.7 & 65.5 \\
\hline & 3200 & $0.905 \pm 0.004$ & $4.521 \pm 0.214$ & $0.800 \pm 0.010$ & $34.9 \pm 3.1$ & $85.1 \pm 1.7$ & $43.4 \pm 5.8$ \\
\hline & 4000 & $0.981 \pm 0.029$ & $3.450 \pm 0.121$ & $0.715 \pm 0.013$ & $359.3 \pm 14.0$ & $97.8 \pm 1.9$ & $81.2 \pm 2.6$ \\
\hline
\end{tabular}

Notes. $t_{\text {evol }}$ - as in Table 1; D.B. - database from which the observed counterpart of filament is selected (F - photographic; V - video-meteor; $\mathrm{R}$ - radio-meteor database); $q$ - perihelion distance; $a$ - semi-major axis; $e$ - eccentricity; $\omega$ - argument of perihelion; $\Omega$-longitude of ascending node; and $i$-inclination to the ecliptic. Quantities $q$ and $a$ are given in astronomical units and angular elements in degrees. 
A\&A 551, A87 (2013)
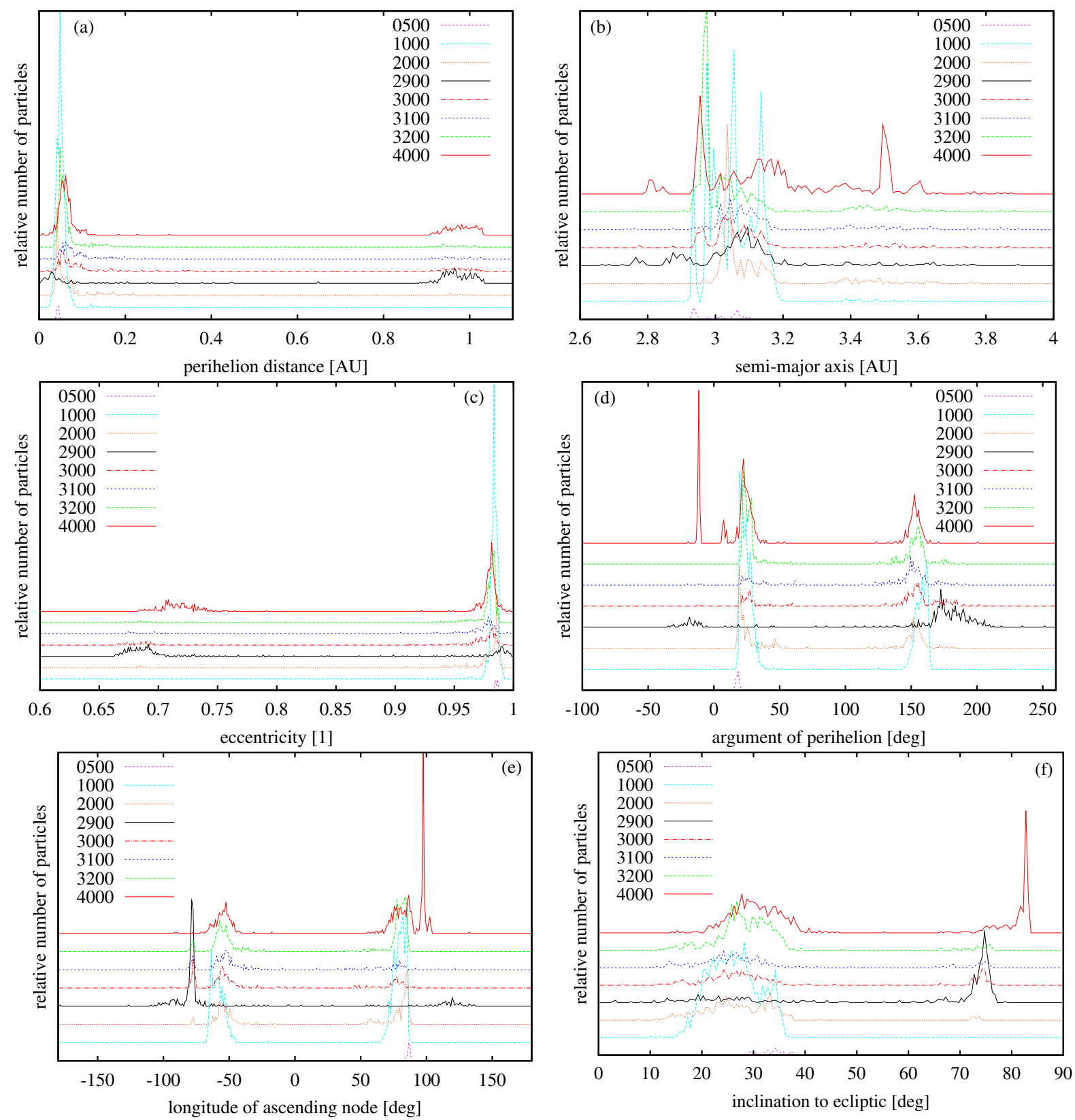

Fig. 4. Distributions of elements of modeled particle orbits approaching the Earth's orbit at a distance shorter than 0.05 AU at present. Specifically, the relative distributions, in arbitrary units, of perihelion distance a), semi-major axis b), eccentricity c), argument of perihelion d), longitude of ascending node e), and inclination to the ecliptic f) are shown for 8 streams modeled 8 times before the present. The times are indicated in the left or right top corner of each plot. For better transparency, the distribution for each successive time is shifted up relatively to the distribution for the preceding time.

filaments 1 and 5. In some relatively short periods, the particles occur mainly in filaments 2,3 , and 4 . During these periods, only a small fraction of particles are moved to filaments 1 and 5 . MT2900 is most likely the latter case. Filament 6 is an exception because it is relatively abundant only in the MT4000 stream.

\subsection{Filament 1: daytime Arietids}

The day-side hemisphere of the Earth is predicted to be struck by the meteoroid particles of the 96P stream in two filaments, which we label in this work as filaments 1 and 4 . The first of these filaments corresponds to the shower that is well-known as daytime Arietids. In more detail, filament 1 is the northern branch of the shower consisting of filaments 1 and 4.
The parameters characterizing the daytime Arietids can only be determined from the members of this shower selected from the radio-meteor database (Table 2). The mean eccentricity of the shower, $0.948 \pm 0.024$, is lower than its predicted counterpart ranging typically from 0.976 to 0.986 for individual modeled streams. The other orbital elements agree with the predicted values within the variation occurring at the modeled streams. The largest differences in angular elements can be seen in the case of the peculiar stream MT2900.

The Arietids were suggested to be related to the two groups of sungrazing comets, Marsden and Kracht. Since only the parabolic approximation of the orbits of these comets is known, it is difficult to evaluate a possible coincidence with our models. Considering the mean argument of perihelion $\left(22.7^{\circ}\right)$, longitude 
L. Neslušan et al.: The meteor-shower complex of 96P/Machholz revisited
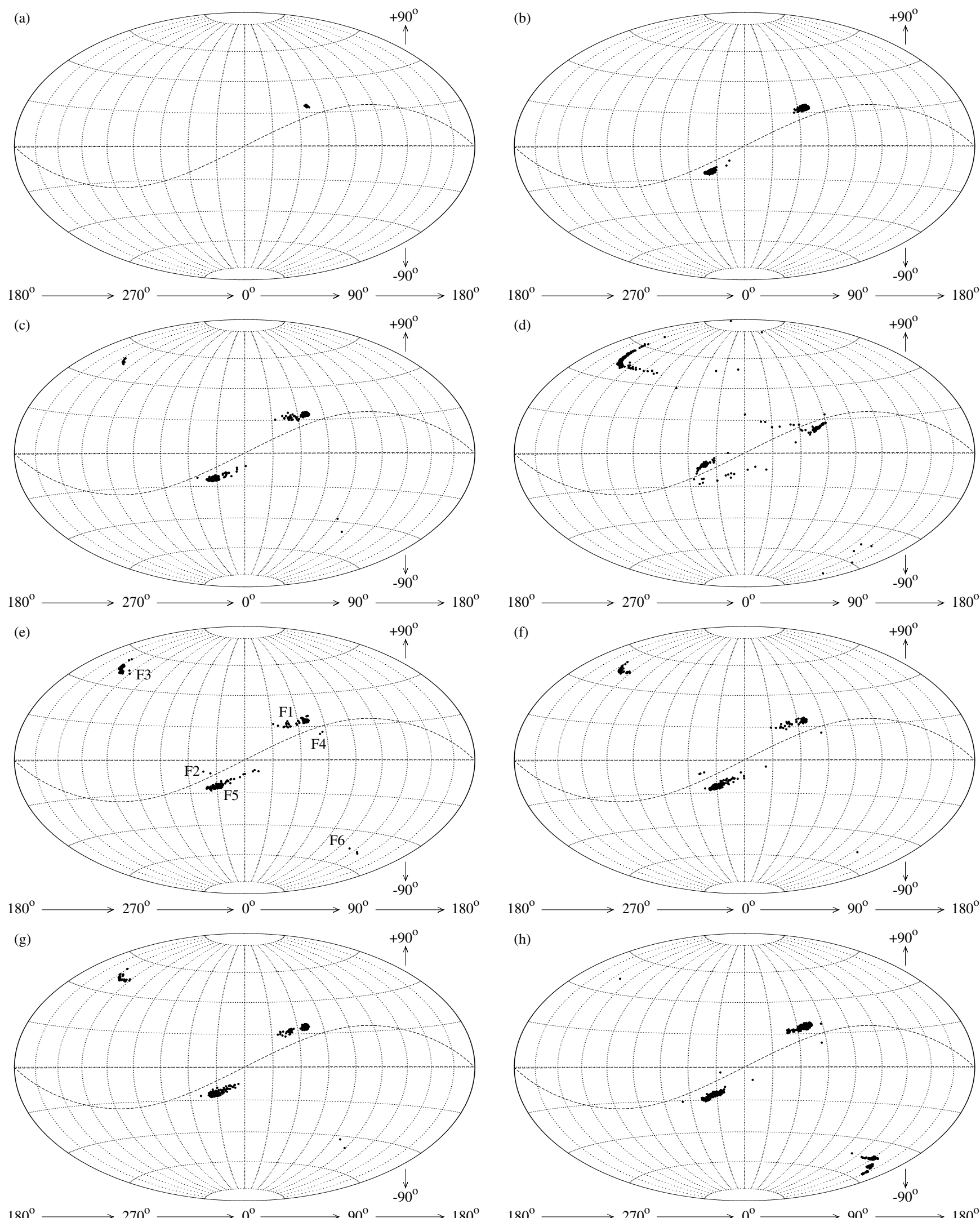

Fig. 5. Positions of radiants of the 96P-stream particles moving currently in orbits, where they can approach the Earth's orbit within 0.05 AU. The positions for the streams MT500, MT1000, MT2000, MT2900, MT3000, MT3100, MT3200, and MT4000 are shown in the plots a)-g), and h), respectively. The stream is split into 6 filaments with 6 distinct radiant areas. The enumeration of the filaments is given in plot e) with the complete set of filamets (F1 to F6). The radiants are shown in the Hammer projection of the sky. The equatorial coordinate frame is used with right ascension indicated by meridional circles and declination indicated with the declination circles. The sinusoid-like curve illustrates the ecliptic. 
of ascending node $\left(81.8^{\circ}\right)$, and inclination $\left(27.0^{\circ}\right)$ determined by Sekanina \& Chodas (2005) for the Marsden group, we can confirm that these values roughly agree with the corresponding values predicted for filament 1 . However, no such confirmation can be stated for the Kracht group with $\left\langle\omega=59.4^{\circ}\right\rangle,\left\langle\Omega=42.8^{\circ}\right\rangle$, and $\left\langle i=13.3^{\circ}\right\rangle$ (Sekanina \& Chodas 2005).

\subsection{Filament 2: $\delta$-Aquarids $N$}

The number of particles of this filament that approach the Earth's orbit is more than five only in the MT2900 stream (35 particles). Nevertheless, the mean orbits of filament 2 of this stream is roughly similar to the orbital parameters of well-known Northern $\delta$-Aquarids found in all three databases of real meteors (Table 2). The real $\delta$-Aquarids $\mathrm{N}$ have a larger perihelion distance and longitude of ascending node (it appears in a later season of the year than predicted), and lower eccentricity and argument of perihelion. These differences seem to exist due to a physical evolution of shower and the consequent selection effect: the particles with a larger perihelion distance are obviously less eroded by solar radiation and solar wind than the extremely sun-grazing particles, therefore the life of these larger- $q$ particles is, on average, relatively longer, and particles can be observed in the Earth's atmosphere with a relatively higher probability.

The above-mentioned hypothesis can be supported by the fact that the larger- $q$ theoretical particles have a larger longitude of ascending node and lower eccentricity and argument of perihelion. For example, the orbital elements $(q, e, \omega, \Omega, i)$ of two particles with the largest $q$ from the stream MT2900 are $\left(0.090 \mathrm{AU}, 0.972,327.7^{\circ}, 133.6^{\circ}, 13.7^{\circ}\right)$ and $(0.075 \mathrm{AU}, 0.976$, $\left.329.0^{\circ}, 132.4^{\circ}, 18.3^{\circ}\right)$, so their orbital elements are closer to those of a real $\delta$-Aquarid $\mathrm{N}$ shower than the average orbital elements of filament 2 .

\subsection{Filament 3: Quadrantids}

The predicted characteristics of this filament fit the well-known major shower well: the Quadrantids. To avoid a misunderstanding, we would like to emphasize that we identify the particles in filament 3 as Quadrantids, but do not suggest that all Quadrantids necessarily originate in comet 96P. Asteroid 2003 EH1 can also be the parent (possibly the main parent) as suggested by Jenniskens (2004). Likewise, it is not excluded that some other objects, like the historical comet C/1490 Y1 (Hasegawa 1979), can be the Quandrantid parent bodies.

The peak in the distribution of $\Omega$ of filament 3 is predicted to be typically narrow, with the values of standard deviation in our models within $1.4^{\circ}$. It corresponds to the well-known short period of activity of Quadrantids. In Table 1, a longer period of the activity is given. However, there is a relatively high peak corresponding to the activity on January 3 and 4. Specifically, $90.0 \%$, $35.6 \%, 67.9 \%, 57.7 \%$, and $56.5 \%$ of all particles in filament 3 approach Earth's orbit only on January 3 to 4 for the streams MT2000, MT2900, MT3000, MT3100, and MT3200, respectively. The corresponding rates of the Quadrantids selected from the photographical and video databases are $93.2 \%$ and $76.2 \%$, respectively.

The good agreement between the predicted and actually observed Quadrantid shower (Tables 1 and 2) implies that comet 96P is certainly as good a Quadrantid parent-body candidate as asteroid $2003 \mathrm{EH} 1$.

In Fig. 5, plot (d), we can see an excursion of some radiants toward a higher declination. Babadzhanov \& Obrubov (1993) assigned this more or less smooth continuation of Quadrantids to another shower: Ursids. We can confirm that the predicted argument of perihelion of high-declination Quadrantids is recognizable higher (consistent with the range given by Babadzhanov \& Obrubov for the Ursids) than that of common Quadrantids. Unfortunately, the small number of particles and vague differences in other parameters do not enable us to draw any serious conclusion about a separate shower. Perhaps, we can classify the Ursids as a "tail" of Quadrantids, which is difficult to separate from the main part of the stream because of the continuity.

\subsection{Filament 4}

A potentially real shower corresponding to filament 4 can be regarded as the southern branch of daytime (Northern) Arietids in analogy to other meteor streams consisting of both northern and southern branches.

The possible existence of the shower is indicated by 42 particles in the case of the MT2900 stream and two particles in the case of the MT3000 stream. (In addition, a single particle of the shower occurs in each of the MT3100 and MT4000 streams.) Therefore, the corresponding real shower, if it exists, can be expected to be very diffuse, with a low value for the zenith hourly rate. Actually, no such shower could be reliably selected from the considered radio database.

The shower would even have a shorter perihelion distance than the Arietids, and only the orbits of relatively old particles could evolve to approach Earth's orbit at present (the particles in filament 4 for the MT2900 stream). These circumstances are unfavorable for the survival of well-populated shower.

However, Babadzhanov \& Obrubov (1993) reported the shower named $\alpha$-Cetids with the observed parameters vaguely consistent with those predicted for filament 4 (Tables 1 and 2), except for a larger declination and geocentric velocity. Specifically, they gave $q=0.06-0.18$ AU, $e=0.89-0.99$, $\omega=194^{\circ}-214^{\circ}, \Omega=255^{\circ}-269^{\circ}, i=20^{\circ}-31^{\circ}, \alpha_{\mathrm{g}}=44^{\circ}-53^{\circ}$, $\delta_{\mathrm{g}}=6^{\circ}-12^{\circ}$, and $V_{\mathrm{g}}=37-39 \mathrm{~km} \mathrm{~s}^{-1}$ for the $\alpha$-Cetids. Therefore, it seems that it is still worthwhile dealing with this part of the $96 \mathrm{P}$ stream.

\subsection{Filament 5: $\delta$-Aquarids $S$}

The mean parameters of this filament are consistent with the mean parameters of the well-known major meteor shower $\delta$-Aquarids $\mathrm{S}$ as determined from individual meteors selected from all three considered databases. In more detail, the values of parameters predicted with the help of models MT1000, MT2000, MT3000, MT3100, MT3200, and MT4000 are slightly shifted with respect to the observed values. However, the corresponding values for the model MT2900 are shifted in the opposite direction, therefore a weighted average from all models tends to fit the observed values better. For example, the geocentric velocity (Table 1) is predicted in the interval from 41.1 to $43.2 \mathrm{~km} \mathrm{~s}^{-1}$ from models MT1000 to MT4000, except for MT2900. This interval is larger than the values of 40.0 and $39.5 \mathrm{~km} \mathrm{~s}^{-1}$ derived from the photographic and video databases, respectively. But the interval is reduced to agree more with these observed values when the value of $36.1 \mathrm{~km} \mathrm{~s}^{-1}$ from the MT2900 model is included in a common average.

Together with the daytime Arietids, Southern $\delta$-Aquarids are the most abundant shower of that part of 96P stream approaching the Earth's orbit. 


\subsection{Filament 6}

The Quadrantids appear to be the northern branch of the toroidal part of the ecliptic-toroidal structure of 96P stream (see a more detailed discussion in Sect. 6). Filament 6 is the southern branch of the toroidal part.

Unfortunately, no real counterpart of filament 6 was found in the databases of observed meteors. Nevertheless, this circumstance can be a consequence of the fact that the radiant area is deep in the southern sky (mean declination of geocentric radiant is, obviously, $\delta_{\mathrm{g}}<-50^{\circ}$ ). In the considered observational data, only a minute fraction of meteors have such radiants. In addition, the angular distance of the mean radiant from the Sun during the shower activity ranges from $\sim 90^{\circ}$ to $\sim 115^{\circ}$; i.e., it is not an unambiguous nighttime shower. The angular distance is similar to that of the Quadrantids. However, the showers are active at the end of December and at the beginning of January (Table 1). While the Quadrantids with the radiants on the northern sky are observed during the long, local-winter nights from the northern hemisphere on Earth, meteors of filament 6 with the radiants on the southern sky can be observed during the short, local-summer nights from the southern hemisphere. Only the observation of radio meteors is not affected by this circumstance.

Babadzhanov \& Obrubov (1993) reported the shower named $\kappa$-Velids with the declination of the mean radiant $\delta_{\mathrm{g}}=-52^{\circ}$ more or less corresponding to a that is predicted for filament 6 . The $\kappa$-Velid right ascension $\left(149^{\circ}\right)$, perihelion distance (0.97 AU), argument of perihelion $\left(18^{\circ}\right)$, and inclination $\left(77^{\circ}\right)$ also roughly agree with the values for filament 6 (Tables 1 and 2), but the observed mean eccentricity of the $\kappa$-Velids is only 0.51 , i.e. apparently lower than the filament- 6 eccentricity.

Jones \& Jones (1993) also found a filament vaguely resembling our filament 6 . They named this hypothetical shower as $\delta$-Velids and predicted its $\left\langle\lambda_{\odot}\right\rangle=270.2^{\circ}, \alpha_{\mathrm{g}}=119.0^{\circ}, \delta_{\mathrm{g}}=$ $-50.7^{\circ}, V_{\mathrm{g}}=36.7 \mathrm{~km} \mathrm{~s}^{-1}$, and the relative strength is an order of magnitude lower than what they predicted for the Quadrantids.

In Fig. 5, plot (h), we can see a structured radiant area of filament 6 in the model MT4000. A more detailed analysis of this structure reveals four separated subareas. Three of these, separated from the fourth by the declination of $-50.5^{\circ}$, have very similar characteristics, therefore can be regarded as a unique (southern) part. The differing mean characteristics of two distinguished parts are, especially, declination $\left(-49.2^{\circ}\right.$ vs. $\left.-55.5^{\circ}\right)$, right ascension $\left(139.5^{\circ}\right.$ vs. $\left.159.3^{\circ}\right)$, geocentric velocity (43.7 vs. $\left.46.5 \mathrm{~km} \mathrm{~s}^{-1}\right)$, argument of perihelion $\left(21.5^{\circ}\right.$ vs. $\left.348.5^{\circ}\right)$, inclination $\left(76.4^{\circ}\right.$ vs. $\left.82.7^{\circ}\right)$, and, perhaps, also perihelion distance (0.958 vs. $0.989 \mathrm{AU})$.

In this context, it is perhaps worth mentioning a possible relationship of the southern substructure with the shower of Carinids reported by Babadzhanov \& Obrubov (1993). These authors characterized the Carinids with $q=0.98 \mathrm{AU}, e=0.61$, $\omega=354^{\circ}, \Omega=108^{\circ}, i=79^{\circ}, \alpha_{\mathrm{g}}=162^{\circ}, \delta_{\mathrm{g}}=-59^{\circ}$, and $V_{\mathrm{g}}=44 \mathrm{~km} \mathrm{~s}^{-1}$.

In the IAU MDC working list of meteor showers ${ }^{2}$, the Carinids are listed as three separated showers: $\alpha$-Carinids, $\eta$-Carinids, and $\epsilon$-Carinids. The above-mentioned southern substructure is approximately consistent only with the $\eta$-Carinids having $\lambda_{\odot}=280.7^{\circ}, \alpha_{\mathrm{g}}=160^{\circ}$, and $\delta_{\mathrm{g}}=-60^{\circ}$ (geocentric velocity is unknown).

\footnotetext{
2 http://www.ta3.sk/IAUC22DB/MDC2007/Roje/roje_lista. php?corobic_roje=2\&sort_roje $=0$
}
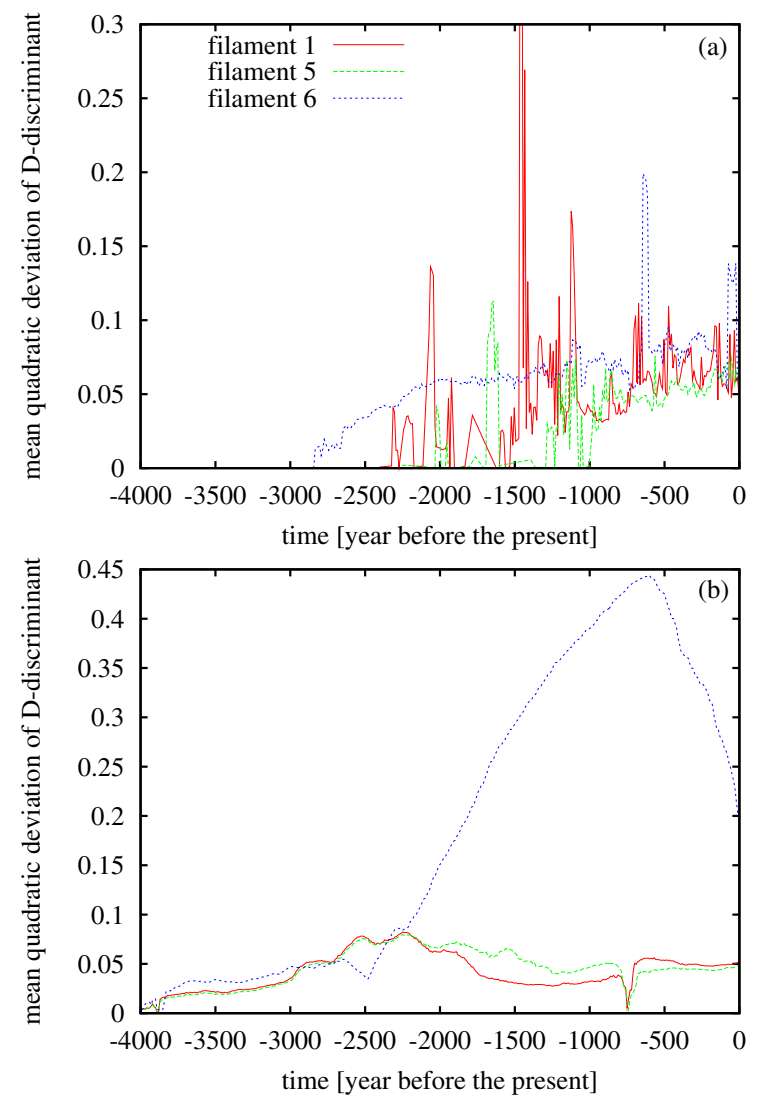

Fig. 6. Evolution of the compactness of abundant filaments 1, 5, and 6 evaluated with the help of the standard deviation, $\sigma$, of the Southworth-Hawkins D-discriminant between the mean orbit of filament and the orbit of each meteor of the filament in given time. While the $\sigma$ of actual filament that approaches Earth's orbit is shown in plot a), the $\sigma$ of selected fixed part of each filament (see end of Sect. 5) is shown in plot b).

At the end of this discussion, we mention the Puppid-Velid Complex and Carinid Complex described by Jenniskens (2006, pp. 516-519). The differences between the presented mean characteristics of these meteor shower groups and filament 6 are relatively small. A larger sample of the data on the complexes collected in the future could, possibly, reveal a relationship between them and the filament of the 96P stream.

\section{Some notes on the aging of filaments}

The age of a meteor stream is usually estimated with the help of the orbit dispersion of its members, which typically increases with time. Using this argument, Jenniskens et al. (1997) concluded that the main component of the Quadrantids is only about 500 years old, since they found a very narrow main peak in the activity curve. However, the perturbation action of Jupiter can cause a more complicated structural evolution of a stream or filament.

We document such an evolution by demonstrating the compactness of the three most abundant filaments 1,5 , and 6 in the MT4000 model. In any given time, we select the members of the given filament according to the above-described radiant areas. The dispersion of orbits in the time can be characterized by the standard deviation, $\sigma$, of the values of Southworth-Hawkins D-discriminant between the orbit of every meteor and mean orbit of the filament. The evolution of this $\sigma$ is shown in Fig. 6 a. 
We can see that there is no linear proportionality between the age and dispersion of filaments. A variation in the perturbation can temporarily increase the dispersion to a strong degree (causing a peak). The periods when the dispersion increases can be temporarily altered by the periods of its decrease. A general trend is similar for all three filaments: the initial steeper increase becomes only moderate, later.

In reality, the abundance of filaments varies with time. Due to the dynamical evolution, some orbits can be deflected from the vicinity of the Earth's orbit. Thus, the dispersion can also change by the most compact core of the filament approaching or going away from the Earth's orbit. To better demonstrate the stabilizing effect of resonance on a filament, we calculate the behavior of another dispersion: that of a "fixed" filament. Specifically, we select a part of each of filaments 1,5 , and 6 , which are the most numerous during the evolution of the actual filament. These and only these particles are regarded as the members of given filament, even if their distance to Earth's orbit remains within $0.05 \mathrm{AU}$ or passes this limit during the evolution. Then we calculate $\sigma$ for this equal-number set of orbits for each output time.

The evolution of the compactness of the fixed filaments is shown in Fig. 6b. From -4000 to about -2300 years, the general evolution of the dispersion is similar for all three filaments: it is slowly increasing. Later, the dispersion of filaments 1 and 5 contrasts with the general rule of stream aging: it decreases until about -750 years and then is nearby constant. The aging of filament 6 is accelerating (the dispersion is higher) in the interval from -2300 years to -600 years before the present, but the dispersion is significantly reduced in subsequent periods, down to about half of the previous maximum.

The abundance of Quadrantids in the MT2900 model, which produces the most shower members at present, is predicted to increase in time about 1500 years before the present until now. In $\sim 500$ years before the present, it was half of the current abundance.

A possible long accumulation of particles in the mean anomaly obviously occurs because the dynamics of the meteoroid complex of the Jupiter-family comet $96 \mathrm{P} / \mathrm{Machholz}$ is largely controlled by Jupiter. This circumstance can cause a synchronization of motion of an essential part of particles with the giant planet and, consequently, the swarm of particles in the given filament might not be distributed uniformly along their mean orbit. Thus, the swarm could cross Earth's orbit just when our planet is not in the place of intersection. The corresponding shower would not exist even though the cloud of particles crosses the orbit of the Earth. We check that this effect occurs at the 96P stream mapping the distribution of particles in the mean anomaly. At any given moment, a variation up to an order of magnitude can actually be found in the distribution. However, there are no empty intervals, the particles are dispersed along the whole interval of mean anomaly. All predicted showers should strike the Earth's atmosphere.

\section{The ecliptic-toroidal structure}

It appears that the meteors come with a significantly higher rate from some specific directions on the sky. The ecliptical streams source from the direction of the Earth's apex, direction toward the Sun named "helion", and the opposite direction named "antihelion". In addition, another two directions were reported from the radar-observation data, situated symmetrically, far from the ecliptic, with the same ecliptic longitude as the Earth's apex: northern and southern "toroidal" directions.
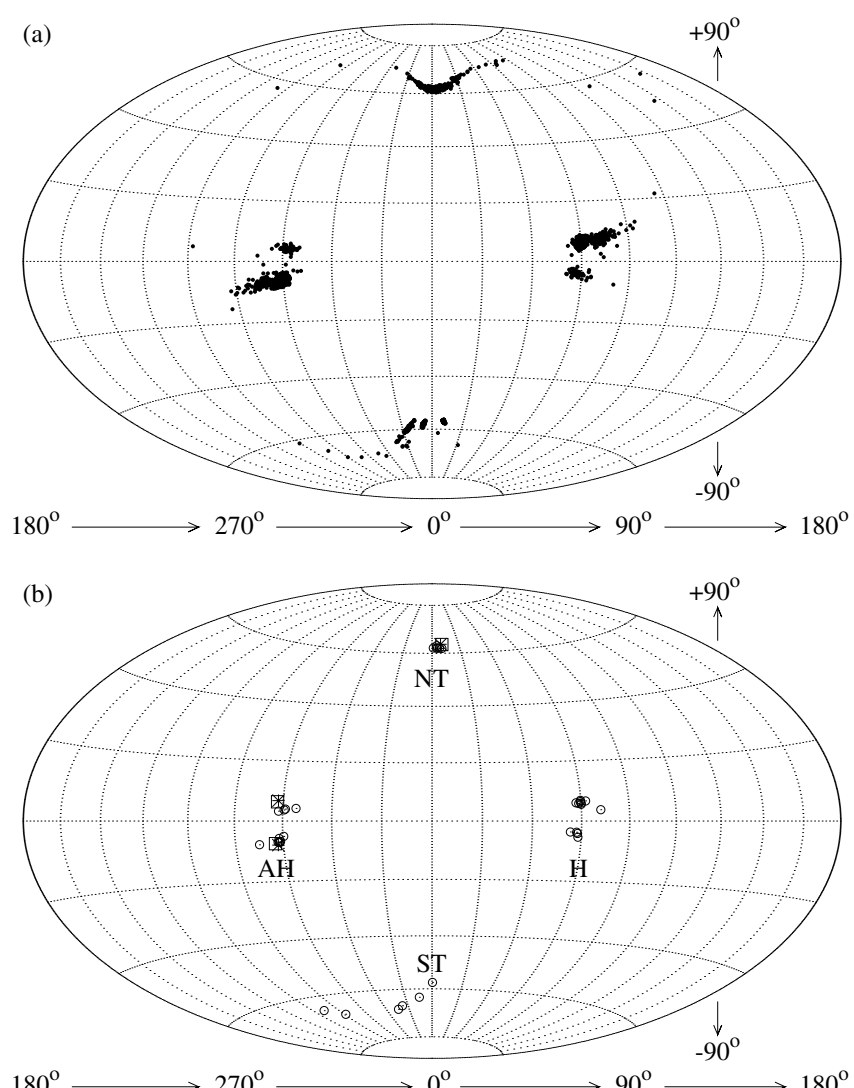

Fig. 7. Ecliptic-toroidal structure of the 96P meteoroid complex. The plots illustrate the positions of radiants in the modified ecliptical coordinate frame $\left(\lambda_{\mathrm{A}}, \beta_{\mathrm{g}}\right)$ in the Hammer projection of celestial sphere. The common ecliptic longitude, $\lambda_{\mathrm{g}}$, is replaced with the angle $\lambda_{\mathrm{A}}$ measured from the ecliptic longitude of the Earth's apex. This apex is situated in the beginning of the frame. Plot a) shows the predicted radiants of theoretical particles in six filaments that approach Earth's orbit, which were found for all eight modeled streams. Plot b) shows the mean radiants of the predicted filaments (empty circles) as well as the mean radiants of real photographic (empty squares) and video (asterices) meteors. NT, ST, H, and AH stand for the northern toroidal, southern toroidal, helion, and antihelion directions, respectively. (See text of Sect. 6.)

The above-described cardinal directions of meteor sources are sometimes related to the sporadic meteor background since the ecliptic-toroidal structure appears in the overall distribution of radio-meteor radiants. A nice distribution showing this structure in reality has recently been published by Campbell-Brown \& Brown (2005; see also Jenniskens 2006, chapt. 28). They used the radiodata from the CMOR (Canadian Meteor Orbit Radar).

According to Jenniskens (2006), many well-known streams, such as Taurids, Daytime Arietids, $\delta$-Aquarids and other belong to the ecliptic streams in sense of cardinal meteor directions. Obviously, there are several parent bodies feeding these streams. In contrast, the toroidal streams are thought to occur due to a bias in radar observations and were found to almost disappear after correcting the bias. Jones et al. (2001) claim that the toroidalstream meteors are merely the high-inclination tail of a continuous distribution of dust from the helion and antihelion sources.

However, our study not only finds that the toroidal streams are real, but also shows a relationship between these and ecliptical streams. To show the ecliptic-toroidal structure transparently, we illustrate the predicted radiants of particles in filaments that approach Earth's orbit and the mean radiants of these filaments in the ecliptical coordinate system modified to place its origin at 
the apex of the Earth's motion (Figs. 7a, and 7b, respectively). In more detail, the ecliptical longitude of the Earth's apex at the moment when the solar longitude is $\lambda_{\odot}$ equals $\lambda_{\odot}+270^{\circ}$. The ecliptic longitude of a radiant measured from the apex, $\lambda_{\mathrm{A}}$, is related to the common ecliptic longitude of the radiant, $\lambda_{\mathrm{g}}$, as

$\lambda_{\mathrm{A}}=\lambda_{\mathrm{g}}-\left(\lambda_{\odot}+270^{\circ}\right)$.

The ecliptic coordinates $\lambda_{\mathrm{g}}, \beta_{\mathrm{g}}$ correspond to the equatorial coordinates $\alpha_{\mathrm{g}}, \delta_{\mathrm{g}}$.

In Fig. 7, we see that our modeling predicts the helion and antihelion, as well as toroidal filaments of the meteor complex originating only in the single parent. All the filaments have the northern and southern branches. The apex part does not occur in this complex.

The ecliptic-toroidal structure of some meteor streams occurs due to the perturbations by Jupiter. We expect that the perturbations forming this structure are efficient at the streams also associated with other parents than comet 96P, which is probably only one of several contributors of the meteoroids to the structure.

\section{Conclusions}

Our analysis of the dynamics of meteoroid particles released from the nucleus of the Jupiter-family comet 96P/1986 J2 (Machholz 1) reveals that the orbits of these particles can evolve into the orbits of several known meteor showers. The dynamical evolution is controlled especially by some systematic perturbations of Jupiter. A large part of the particles resides, for a quite long periods, in well-defined orbital corridors.

The 96P stream approaches the Earth's orbit in six intervals of the ecliptic longitude of Earth. Two of these intervals partially overlap. Since any given interval is situated close to another, we can speak about three close pairs of the intervals. The particles in three of these intervals hit the Earth from the northern direction and in the other three from the southern direction relative to the ecliptic. As a consequence, we can distinguish six filaments in the part of the stream that approaches the orbit of Earth. These filaments correspond to six showers. Four of them are well known from observations: daytime Arietids, $\delta$-Aquarids $\mathrm{S}$ and N, and Quadrantids.

Both branches of $\delta$-Aquarids, daytime Arietids, and one weak shower (identified by Babadzhanov \& Obrubov (1993) with the $\alpha$-Cetids) constitute the ecliptical substructure of the complex. The Quadrantids and another shower with the radiant situated deeply in the southern sky, so almost not observed until now, constitute the toroidal substructure of the 96P complex. We would like to point out the direct relationship between the ecliptical and toroidal showers, which evolve into the observed orbits from the similar initial orbits situated in a common orbital corridor around the orbit of the parent body.

Since the motion of the particles in the 96P stream is controlled by Jupiter, their orbits often librate and, consequently, the dispersion of orbits in the filaments does not increase monotonously in time. Therefore the argument of small or large observed dispersion cannot be used to determine the age of any filament of the 96P-complex structure. The libration also causes a variation in the rate of meteors in the showers over centuries or millennia.

The meteoroid complex of $96 \mathrm{P}$ can be replenished with meteoroids by several other bodies, not only by $96 \mathrm{P}$ itself. In earlier studies (e.g. Williams et al. 2004; Porubčan \& Kornoš 2005; Ryabova \& Nogami 2005; Wiegert \& Brown 2005; Kaňuchová $\&$ Neslušan 2007), a close similarity between the orbits of both comet 96P and asteroid $2003 \mathrm{EH} 1$ in some periods was found. Therefore, this asteroid, regarded as the parent body (or one of parent bodies) of Quadrantids, also belongs to the complex. Likewise, other candidates for the Quadrantid parent can be the complex members. Besides the historical comets C/1490 Y1 and C/1385 U1 (Hasegawa 1979), asteroid 5496 (1973 NA) (Williams \& Collander-Brown 1998) as well as comets D/1783 W1 (Pigott), 5D/1846 D2 (Brorsen), D/1892 T1 (Barnard 3), and P/1994 P1 (Machholz 2) and asteroids 1994 JX, 1999 LT1, 2000 PG3, 2002 AR129, 2002 KF4, 2002 UO3, 2003 YS1, and 2004 BZ74 (Wiegert \& Brown 2005) have been suggested as contributors to the Quadrantids.

A relationship between the sungrazing comets of the Marsden and Kracht groups and the daytime Arietids has been considered from the time these groups were discovered. Therefore, these comets could also be members of the 96P complex. Unfortunately, only the parabolic approximation of their orbits is known, therefore it is hard to draw serious conclusions about the actual relation between the comets of these groups and the 96P complex. Considering their mean orbits derived by Sekanina \& Chodas (2005), we can claim some similarity between the Marsden group and our prediction of the mean angular elements for daytime Arietids. However, a relationship of the Kracht group with any filament of $96 \mathrm{P}$ complex is questionable.

According to our analysis, the orbit of any object, which is situated within a filament of the 96P-complex structure, can be changed into an orbit of any other filament of this structure. If we modeled the meteoroid stream for any comet or asteroid, which is in one of filaments of the 96P complex, we could expect a similar structure to the modeled streams of 96P. Since we studied the stream of comet $96 \mathrm{P} / \mathrm{Machholz}$, we called the complex by this name, but it is still uncertain if this comet alone is the main contributor to the complex, hence its main parent body.

We can conclude that comet 96P, with the meteoroids supplies mainly the $\delta$-Aquarid S and daytime Arietid filaments. The particles start to approach the Earth's orbit in orbits of daytime Arietids and $\delta$-Aquarids S soon after their escape from the comet nucleus, because of the smallest change in orbits needed. This is obviously also why only these filaments are so abundant. Only later do the particles enter the corridors of other filaments, but obviously in lower numbers as their orbits have to evolve more. Modeling for time 500 years before the present yields $0.4 \%$ of all modeled particles in the Arietid filament and no particle in the other filaments at present. For the other models, the abundance of the Arietid and $\delta$-Aquarid $\mathrm{S}$ filaments varies from $0.05 \%$ to $9.2 \%$ and from $0.1 \%$ to $6.8 \%$, respectively.

The abundances of filament 3 (Quadrantids) are typically about one order of magnitude smaller (below 1\%) than those of filaments 1 and 5. The Quadrantid filament reaches the maximum abundance for MT2900 model (2.7\% of all modeled particles). Its southern toroidal counterpart, filament 6 , reaches the maximum abundance for MT4000 model (2.6\%). For the other models, its abundance is less than $0.05 \%$. The occurrence of particles in filaments 2 and 4 is only indicative in our models (less than $0.5 \%$ ). The orbital planes of these particles have to "cross" the orbital plane of Jupiter. Obviously, the low number is caused by the large perturbations during this event.

An estimate of the relative rate of contributions to the $96 \mathrm{P}$ stream by $96 \mathrm{P}$ itself and other possible parents is complicated by two problems. First, our knowledge of daytime Arietids is based on the less precise radio observations. In addition, the available radio database of meteors is not homogeneous, therefore a good comparison among the rates of Arietids and other 96P-complex showers is impossible. Second, there is a lack of 
data for meteors with the radiants on the southern sky. Although a filament 6 ( $\kappa$-Velids?), with the declination of radiant lower than $-50^{\circ}$, is predicted to be relatively abundant, we cannot compare our prediction with reality. Perhaps, this problem will soon be removed with the help of photographic data produced by the newly installed fireball network in southwest Australia (Bland et al. 2005, 2008).

Acknowledgements. This article was supported by the realization of the Project ITMS No. 26220120029, based on the supporting operational Research and development program financed from the European Regional Development Fund. The work was also supported, in part, by the VEGA - the Slovak Grant Agency for Science, grants No. 0011 (L.N. \& D.T.) and 0022 (Z.K.).

\section{References}

Astronomical Almanac 2004. 2002, Washington: US Government Printing Office and London: The Stationery Office

Astronomical Almanac 2005. 2003, Washington: US Government Printing Office and London: The Stationery Office

Astronomical Almanac 2006. 2004, Washington: US Government Printing Office and London: The Stationery Office

Astronomical Almanac 2008. 2006, Washington: US Government Printing Office and London: The Stationery Office

Babadzhanov, P. B., \& Obrubov, Yu. V. 1987, in Interplanetary Matter, Proc. 10-th ERAM, eds. Z. Ceplecha, \& P. Pecina, Astron. Inst. Czechosl. Acad. Sci., Ondřejov, 141

Babadzhanov, P. B., Obrubov, Yu. V., \& Pushkarev, A. N. 1991, Astron. Vestn., 25, 82 (in Russian)

Babadzhanov, P. B., \& Obrubov, Yu. V. 1992, in Asteroids, Comets, Meteors 1991 (Lunar and Planetary Inst., Houston), 27

Babadzhanov, P. B., \& Obrubov, Yu. V. 1993, in Meteoroids and Their Parent Bodies, eds. J. Štohl, \& I. P. Williams, Astron. Inst., Slovak Acad. Sci., Bratislava, 49

Bland, P. A., Spurný, P., Bevan, A. W. R., et al. 2005, Meteorit. Planet. Sci. Suppl., 40, 5225

Bland, P. A., Spurný, P., Shrbený, L., et al. 2008, LPI Contribution, 1405, 8246 Campbell-Brown, M. D., \& Brown, P. 2005, in Workshop on Dust in Planetary Systems - abstracts, September 26-30, Kauai, Hawaii

Chambers, J. E. 1999, MNRAS, 304, 793

Chernitsov, A. M., Baturin, A. P., \& Tamarov V. A. 1998, Sol. Syst. Res., 32, 405

Everhart, E. 1985, in Dynamics of Comets: Their Origin and Evolution, eds. A. Carusi, \& G. B. Valsecchi (Dordrecht: Kluwer), 185

Froeschlé, C., \& Scholl, H. 1986, A\&A, 158, 259
Froeschlé, C., Gonczi, R., \& Rickman, H. 1993, in Meteoroids and Their Parent Bodies, eds. J. Štohl, \& I. P. Williams, Astron. Inst., Slovak Acad. Sci., Bratislava, 169

Gonczi, R., Rickman, H., \& Froeschlé, C. 1992, MNRAS, 254, 627

Hasegawa, I. 1979, Publ. Astron. Soc. Japan, 31, 257

Hawkins, G. S. 1963, Smithsonian Contr. Astrophys., 7, 53

Hughes, D. W., Williams, I. P., \& Murray, C. D. 1979, MNRAS, 189, 493

Jenniskens, P. 2004, AJ, 127, 3018

Jenniskens, P. 2006, Meteor Showers and Their Parent Comets (Cambridge: Cambridge Univ. Press)

Jenniskens, P., Betlem, H., de Lignie, M., Langbroek, M., \& van Vliet, M. 1997, A\&A, 327, 1242

Jones, J., \& Jones, W. 1993, MNRAS, 261, 605

Jones, J., Campbell, M., \& Nikolova, S. 2001, in Proceedings of the Meteoroids, ESA SP 495, ed. B. Warmbein (Noordwijk: ESA Publications Division), 575 Kaňuchová, Z., \& Neslušan, L. 2007, A\&A, 470, 1123

Ki-Won, L., Hong-Jin, Y., \& Myeong-Gu, P. 2009, MNRAS, 400, 1389

Lindblad, B. A., Neslušan, L., Porubčan, V., \& Svoreň, J. 2003, Earth, Moon, Planets, 93, 249

Marsden, B. G. 1989, Catalogue of Cometary Orbits, 6-th edn. (Cambridge: Smithsonian Astrophys. Obs.)

McIntosh, B. A. 1990, Icarus, 86, 299

Micheli, M., Bernardi, F., \& Tholen, D. J. 2008, MNRAS, 390, L6

Milani, A. 1999, Icarus, 137, 269

Milani, A., Sansaturio, M. E., Tommei, G., Arratia, O., \& Chesley, S. R. 2005, A\&A, 431, 729

Neslušan, L. 1999, A\&A, 351, 752

Neslušan, L., Svoreň, J., \& Porubčan, V. 1995, Earth, Moon, Planets, 68, 427

Neslušan, L., Svoreň, J., \& Porubčan, V. 2012, Earth, Moon, Planets, submitted Porubčan, V., \& Kornoš, L. 2005, Contrib. Astron. Obs. Skalnaté Pleso, 35, 5

Ryabova, G., \& Nogami, N. 2005, in Proc. Internat. Meteor Conference held at Varna, Bulgaria, 23-26 September, 2004, eds. M. Triglav-Čekada, J. Kac, \& A. McBeath, International Meteor Organization, 63

Sekanina, Z., \& Chodas, P. W. 2005, ApJ, 161, 551

Sekanina, Z., \& Southworth, R. B. 1975, Physical and dynamical studies of meteors. Meteor-fragmentation and stream-distribution studies. Final Report, Cambridge, MA: Smithsonian Astrophys. Obs.

SonotaCo. 2009, WGN - Journal of the IMO, 37, 55 http: //sonotaco.jp/doc/SNM/

Tomko, D., \& Neslušan, L. 2012, Earth, Moon, Planets, 108, 123

Wiegert, P., \& Brown P. 2005, Icarus, 179, 139

Williams, I. P., \& Wu, Z. 1993, MNRAS, 264, 659

Williams, I. P., Murray, C. D., \& Hughes, D. W. 1979, MNRAS, 189, 483

Williams, I. P., \& Collander-Brown, S. J. 1998, MNRAS, 294, 127

Williams, I. P., Ryabova, G. O., Baturin, A. P., \& Chernitsov, A. M. 2004, MNRAS, 355, 1171

Wu, Z., \& Williams, I. P. 1992, MNRAS, 259, 617 